\title{
On function spaces of Lorentz-Sobolev type
}

\author{
Blanca F. Besoy • Fernando Cobos • Hans \\ Triebel
}

Received: date / Accepted: date

\begin{abstract}
We work with Triebel-Lizorkin spaces $F_{q}^{s} L_{p, r}\left(\mathbb{R}^{n}\right)$ and Besov spaces $B_{q}^{s} L_{p, r}\left(\mathbb{R}^{n}\right)$ with Lorentz smoothness. Using their characterizations by real interpolation we show how to transfer a number of properties of the usual Triebel-Lizorkin and Besov spaces to the spaces with Lorentz smoothness. In particular, we give results on diffeomorphisms, extension operators, multipliers and we also show sufficient conditions on parameters for $F_{q}^{s} L_{p, r}\left(\mathbb{R}^{n}\right)$ and $B_{q}^{s} L_{p, r}\left(\mathbb{R}^{n}\right)$ to be multiplication algebras.
\end{abstract}

Keywords Triebel-Lizorkin-Lorentz spaces $\cdot$ Besov-Lorentz spaces $\cdot$ real interpolation $\cdot$ multiplications

Mathematics Subject Classification (2010) Primary 46E35, 46M35 - Secondary 42C $40,42 \mathrm{~B} 35$

B. F. Besoy and F. Cobos have been partially supported by Ministerio de Ciencia e Innovación MTM201784058-P(AEI/FEDER, UE).

B. F. Besoy has also been supported by FPU grant FPU 16/02420 of the Spanish Ministerio de Educación, Cultura y Deporte.

Blanca F. Besoy

Departamento de Análisis Matemático y Matemática Aplicada, Facultad de Matemáticas, Universidad Complutense de Madrid. Plaza de Ciencias 3, 28040 Madrid, Spain.

Current address: Departamento de Ingeniería Geológica y Minera, Escuela Técnica Superior de Ingenieros de Minas y Energía, Universidad Politécnica de Madrid, Calle de Alenza 4, 28003 Madrid. Spain.

E-mail: blanca.f.besoy@ucm.es E-mail: blanca.fbesoy@upm.es

Fernando Cobos (Corresponding author) - ORCID ID: 0000-0001-9059-4356

Departamento de Análisis Matemático y Matemática Aplicada, Facultad de Matemáticas, Universidad Complutense de Madrid. Plaza de Ciencias 3, 28040 Madrid, Spain.

E-mail: cobos@mat.ucm.es

Hans Triebel

Institut für Mathematik, Friedrich-Schiller-Universität. Ernst-Abbe-Platz 2, 07737 Jena, Germany.

E-mail: hans.triebel@uni-jena.de 


\section{Introduction}

In the theory of function spaces, a central role is played by the scales of TriebelLizorkin spaces $F_{p, q}^{s}\left(\mathbb{R}^{n}\right)$ and of Besov spaces $B_{p, q}^{s}\left(\mathbb{R}^{n}\right)$. These families include many well-known spaces of functions and distributions as Sobolev spaces, fractional Sobolev spaces (= Bessel potential spaces), Hölder-Zygmund spaces, local Hardy spaces or Lebesgue spaces (see the books by Triebel $[48-50,55,56]$ ). Among other things, on spaces $A_{p, q}^{s}\left(\mathbb{R}^{n}\right), A \in\{B, F\}$, rely a substantial part of the recent theory of Navier-Stokes equations and of distinguished PDE models for hydrodynamics and chemotaxis.

Several refinements of these scales have been also studied in the literature. They are motivated either by applications to concrete questions or to complete the description of certain properties of the spaces $A_{p, q}^{s}\left(\mathbb{R}^{n}\right)$. This is the case of the function spaces with Lorentz smoothness $A_{q}^{s} L_{p, r}\left(\mathbb{R}^{n}\right), A \in\{B, F\}$.

In the monograph of Triebel [47, Sections 2.4.1 and 2.4.2] one can find the most relevant interpolation properties of the spaces $A_{p, q}^{s}\left(\mathbb{R}^{n}\right)$ for $1 \leq p, q \leq \infty$. If $p_{1} \neq p_{2}$, applying the real method to the couple $\left(A_{p_{1}, q}^{s}\left(\mathbb{R}^{n}\right), A_{p_{2}, q}^{s}\left(\mathbb{R}^{n}\right)\right)$, it turns out that the resulting space does not belong to the scale of Triebel-Lizorkin spaces or the scale of Besov spaces. To describe them one needs to refine these scales. Specifically, in the Fourier-analytical definition of $A_{p, q}^{s}\left(\mathbb{R}^{n}\right)$, one should replace the Lebesgue space $L_{p}\left(\mathbb{R}^{n}\right)$ by the more general Lorentz space $L_{p, r}\left(\mathbb{R}^{n}\right)$ (see [47, Theorems $2.4 .1 /(\mathrm{c})$ and $2.4 .2 / 1$, (c)]). In this way the spaces with Lorentz smoothness $A_{q}^{s} L_{p, r}\left(\mathbb{R}^{n}\right)$ arise.

Since the early 1960s spaces with Lorentz smoothness have been used repeatedly in the literature. For example, Hardy-Lorentz spaces have been used by Fefferman, Riviere and Sagher [19] and Almeida and Caetano [2,3], Bessel-Lorentz potential spaces $F_{2}^{s} L_{p, r}\left(\mathbb{R}^{n}\right)$ appear in the papers by Stein [45], Caetano [9], Cianchi and Pick [11], Ko and Lee [31] and Grafakos and Slavíková [24]. Spaces $F_{q}^{s} L_{p, \infty}\left(\mathbb{R}^{n}\right)$ and $B_{q}^{s} L_{p, \infty}\left(\mathbb{R}^{n}\right)$ (weak- $A_{p, q}^{s}\left(\mathbb{R}^{n}\right)$ spaces) appear in the book by Edmunds and Triebel [18]. Yang, Cheng and Peng [59] studied Lorentz smoothness spaces in connection with wavelet theory. Triebel-Lizorkin-Lorentz spaces in the context of partial differential equations have been considered by Xiang and Yan [57,58]. More recently, Seeger and Trebels [43] have characterized all embeddings between spaces $A_{q}^{s} L_{p, r}\left(\mathbb{R}^{n}\right)$, and Hobus and Saal [25] have applied the spaces $F_{q}^{s} L_{p, r}\left(\mathbb{R}^{n}\right)$ to study Navier-Stokes equations.

Our aim in this paper is to continue the research on basic properties of spaces with Lorentz smoothness. Using the interpolation properties of the spaces $F_{q}^{s} L_{p, r}\left(\mathbb{R}^{n}\right)$ and $B_{q}^{s} L_{p, r}\left(\mathbb{R}^{n}\right)$, we are going to transfer a number of properties of the usual TriebelLizorkin and Besov spaces to the spaces with Lorentz smoothness. In particular, we study three of the so-called key problems in [49, Chapter 4]: Invariance of $A_{q}^{s} L_{p, r}\left(\mathbb{R}^{n}\right)$ with respect to diffeomorphisms of $\mathbb{R}^{n}$ onto itself, the existence of linear extension operators of corresponding spaces $A_{q}^{s} L_{p, r}\left(\mathbb{R}_{+}^{n}\right)$ on $\mathbb{R}_{+}^{n}$ to $A_{q}^{s} L_{p, r}\left(\mathbb{R}^{n}\right)$ and pointwise multipliers. The other problem mentioned in [49], i.e. traces of $A_{q}^{s} L_{p, r}\left(\mathbb{R}^{n}\right)$ on hyperplanes, is discussed in [7]. We also establish some multiplication results on the spaces $A_{q}^{s} L_{p, r}\left(\mathbb{R}^{n}\right)$. In particular, we show sufficient conditions on the parameters for $F_{q}^{s} L_{p, r}\left(\mathbb{R}^{n}\right)$ to be a multiplication algebra. For spaces $B_{q}^{s} L_{p, r}\left(\mathbb{R}^{n}\right)$ we establish the corresponding results. 
The paper is organized as follows. In Section 2 we review some basic results from real interpolation theory of quasi-Banach couples. Triebel-Lizorkin-Lorentz spaces are introduced in Section 3, where we also characterize them by interpolation. In Section 4 we establish several properties of spaces $F_{q}^{s} L_{p, r}\left(\mathbb{R}^{n}\right)$. We describe the smoothing effect of the Gauss-Weierstrass semi-group, we give a result on Fourier multipliers and the results on diffeomorphisms, extension operators and smooth multipliers. Multiplication properties of the spaces $F_{q}^{s} L_{p, r}\left(\mathbb{R}^{n}\right)$ are discussed in Section 5. Besov-Lorentz spaces are introduced in Section 6, where we show that they coincide with Besov spaces modelled on local Hardy-Lorentz spaces, studied by Almeida and Caetano [3]. For this aim we establish a Fourier multiplier result for Lorentz spaces. Finally, in Section 7, we describe several properties of spaces $B_{q}^{s} L_{p, r}\left(\mathbb{R}^{n}\right)$. We give there results on diffeomorphisms, extension operators, smooth multipliers and sufficient conditions on $B_{q}^{s} L_{p, r}\left(\mathbb{R}^{n}\right)$ to be a multiplication algebra.

\section{Real interpolation}

Subsequently, given an arbitrary index set $I$ and two sets of positive numbers $\left\{a_{i}: i \in\right.$ $I\}$ and $\left\{b_{i}: i \in I\right\}$, we write $a_{i} \lesssim b_{i}$ if there is a positive number $c$ such that $a_{i} \leq c b_{i}$ for all $i \in I$. We put $a_{i} \sim b_{i}$ if $a_{i} \lesssim b_{i}$ and $b_{i} \lesssim a_{i}$.

Let $(A,\|\cdot \mid A\|)$ be a complex quasi-Banach space with constant $c=c_{A} \geq 1$ in the quasi-triangle inequality. Let $0<p \leq 1$ be such that $2^{1 / p-1}=c$. According to the Aoki-Rolewicz theorem, there is another quasi-norm $\|\cdot\|_{*}$ on $A$ which is equivalent to $\|\cdot \mid A\|$ and satisfies that

$$
\left\|a_{1}+a_{2}\right\|_{*}^{p} \leq\left\|a_{1}\right\|_{*}^{p}+\left\|a_{2}\right\|_{*}^{p}, a_{1}, a_{2} \in A
$$

(see [4], [39], [32, §15.10] or [38, pp. 92-93]). We say that $\|\cdot\|_{*}$ is a $p$-norm and that $A$ is a $p$-Banach space. Note that if $0<r<p$ then $A$ is also an $r$-Banach space.

If $A$ and $B$ are quasi-Banach spaces, by $A \hookrightarrow B$ we mean that $A$ is continuously embedded in $B$.

Examples of quasi-Banach spaces are the usual spaces of $q$-summable scalar sequences $\ell_{q}, 0<q \leq \infty$. For $1 \leq q \leq \infty$ then $\ell_{q}$ is a Banach space and so it is an 1 -Banach space. If $0<q<1$ then $\ell_{q}$ is $p$-Banach if, and only if, $0<p \leq q$. Indeed, as far as the latter is concerned, assume that $\|\cdot\|_{*}$ is an equivalent $p$-norm in $\ell_{q}$. In particular, $\left\|e_{j}\right\|_{*} \sim 1$ where $e_{j}$ is the unit element with 1 at place $j$ and zero otherwise. Let $a=\sum_{j=1}^{r} \lambda_{j} e_{j}$ with $\lambda_{j} \in \mathbb{C}$ and $r \in \mathbb{N}$. Then

$$
\begin{aligned}
\left(\sum_{j=1}^{r}\left|\lambda_{j}\right|^{q}\right)^{1 / q} & =\left\|\sum_{j=1}^{r} \lambda_{j} e_{j} \mid e_{q}\right\| \sim\left\|\sum_{j=1}^{r} \lambda_{j} e_{j}\right\|_{*} \\
& \leq\left(\sum_{j=1}^{r}\left|\lambda_{j}\right|^{p}\left\|e_{j}\right\|_{*}^{p}\right)^{1 / p} \leq c\left(\sum_{j=1}^{r}\left|\lambda_{j}\right|^{p}\right)^{1 / p} .
\end{aligned}
$$

This requires $p \leq q$.

By a quasi-Banach (respectively, $p$-Banach) couple $\left(A_{1}, A_{2}\right)$ we mean two quasiBanach spaces (respectively, two $p$-Banach spaces) $A_{1}, A_{2}$ which are continuously embedded in the same Hausdorff topological vector space $\mathcal{A}$. 
The Peetre's $K$-functional is defined by

$$
K(t, a)=K\left(t, a ; A_{1}, A_{2}\right)=\inf \left\{\left\|a_{1}\left|A_{1}\|+t\| a_{2}\right| A_{2}\right\|: a=a_{1}+a_{2}, a_{j} \in A_{j}\right\},
$$

$a \in A_{1}+A_{2}$ and $t>0$. Note that $K(t, \cdot)$ is a quasi-norm in $A_{1}+A_{2}$ and that the quasi-triangle inequality holds with the same constant $c=\max \left(c_{A_{1}}, c_{A_{2}}\right) \geq 1$ for any $t>0$.

If $\left\|\cdot \mid A_{j}\right\|$ is a $p$-norm, $j=1,2$, then it is useful to consider the functional

$$
K_{p}(t, a)=\inf \left\{\left(\left\|a_{1}\left|A_{1}\left\|^{p}+t^{p}\right\| a_{2}\right| A_{2}\right\|^{p}\right)^{1 / p}: a=a_{1}+a_{2}, a_{j} \in A_{j}\right\}
$$

which is a $p$-norm in $A_{1}+A_{2}$ and satisfies that

$$
K(t, a) \leq K_{p}(t, a) \leq 2^{1 / p-1} K(t, a), a \in A_{1}+A_{2}, t>0 .
$$

For $0<\theta<1$ and $0<q \leq \infty$, the real interpolation space $\left(A_{1}, A_{2}\right)_{\theta, q}$ consists of all $a \in A_{1}+A_{2}$ having a finite quasi-norm

$$
\left\|a \mid\left(A_{1}, A_{2}\right)_{\theta, q}\right\|= \begin{cases}\left(\int_{0}^{\infty}\left(t^{-\theta} K(t, a)\right)^{q} \frac{d t}{t}\right)^{1 / q} & \text { if } q<\infty \\ \sup _{t>0}\left\{t^{-\theta} K(t, a)\right\} & \text { if } q=\infty .\end{cases}
$$

The space $\left(A_{1}, A_{2}\right)_{\theta, q}$ is a Banach space provided $1 \leq q \leq \infty$ and that $\left(A_{1}, A_{2}\right)$ is a Banach couple. Otherwise, it is a quasi-Banach space. If $q<\infty$ then $A_{1} \cap A_{2}$ is dense in $\left(A_{1}, A_{2}\right)_{\theta, q}$. We refer to the books by Bergh and Löfström [6], Triebel [47] and Bennett and Sharpley [5] for other properties of real interpolation spaces. Among them we have the following interpolation property for bounded linear operators: Let $\left(B_{1}, B_{2}\right)$ be another quasi-Banach couple. If $T$ is a linear operator such that $T: A_{j} \rightarrow B_{j}$ is bounded for $j=1,2$, then $T:\left(A_{1}, A_{2}\right)_{\theta, q} \rightarrow\left(B_{1}, B_{2}\right)_{\theta, q}$ is also bounded with $\|T\|_{\left(A_{1}, A_{2}\right)_{\theta, q},\left(B_{1}, B_{2}\right)_{\theta, q}} \leq\|T\|_{A_{1}, B_{1}}^{1-\theta}\|T\|_{A_{2}, B_{2}}^{\theta}$.

Lemma 2.1 Let $\left(A_{1}, A_{2}\right)$ be a p-Banach couple, $0<p \leq 1$. Let $0<\theta<1$ and $0<q \leq \infty$. Then the space $\left(A_{1}, A_{2}\right)_{\theta, q}$ is $\min (p, q)$-Banach.

Proof Consider the quasi-norm $\|\cdot\|_{\theta, q}$ in $\left(A_{1}, A_{2}\right)_{\theta, q}$ which is obtained by replacing the $K$-functional by $K_{p}(t, a)$ in the definition of $\left\|\cdot \mid\left(A_{1}, A_{2}\right)_{\theta, q}\right\|$. By (2.1) we have that $\left\|\cdot \mid\left(A_{1}, A_{2}\right)_{\theta, q}\right\| \sim\|\cdot\|_{\theta, q}$. If $x_{1}, x_{2} \in A_{1}+A_{2}$, we get

$$
\left\|x_{1}+x_{2}\right\|_{\theta, q} \leq\left(\int_{0}^{\infty} t^{-\theta q}\left(K_{p}\left(t, x_{1}\right)^{p}+K_{p}\left(t, x_{2}\right)^{p}\right)^{q / p} \frac{d t}{t}\right)^{1 / q}
$$

(with standard modifications when $q=\infty$ ). If $q \geq p$ then it follows from the triangle inequality of $L_{q / p}(0, \infty)$ that

$$
\left\|x_{1}+x_{2}\right\|_{\theta, q}^{p} \leq \sum_{j=1}^{2}\left(\int_{0}^{\infty}\left(t^{-\theta p} K_{p}\left(t, x_{j}\right)^{p}\right)^{q / p} \frac{d t}{t}\right)^{p / q}=\left\|x_{1}\right\|_{\theta, q}^{p}+\left\|x_{2}\right\|_{\theta, q}^{p} .
$$


Hence, $\left(A_{1}, A_{2}\right)_{\theta, q}$ is $p$-normed. If $0<q<p \leq 1$ then $A_{1}$ and $A_{2}$ are also $q$-normed spaces. Using this time $K_{q}(t, a)$ to define $\|\cdot\|_{\theta, q}$, we obtain

$$
\left\|x_{1}+x_{2}\right\|_{\theta, q}^{q} \leq \sum_{j=1}^{2} \int_{0}^{\infty} t^{-\theta q} K_{q}\left(t, x_{j}\right)^{q} \frac{d t}{t}=\left\|x_{1}\right\|_{\theta, q}^{q}+\left\|x_{2}\right\|_{\theta, q}^{q} .
$$

This shows that $\left(A_{1}, A_{2}\right)_{\theta, q}$ is a $q$-Banach space.

In general, the best possible $\rho$ with $0<\rho \leq 1$ for which $\left(A_{1}, A_{2}\right)_{\theta, q}$ is a $\rho$-Banach space is the value given in Lemma 2.1. We will show it later in Example 2.3.

Bounded bilinear operators can also be interpolated by the real method. For Banach couples, this question was already considered in the seminal paper of Lions and Peetre [35] on the real method. The extension to quasi-Banach couples was studied by Karadzhov [30] and König [33]. See also the papers by Fernández-Cabrera and Martínez [20] and Cobos, Fernández-Cabrera and Martínez [14] for results on interpolation methods which extend the real method. In particular, proceeding as in [20, Theorem 4.1] and [14, Theorem 3.1] one can derive the following result.

Theorem 2.2 Let $\left(A_{1}, A_{2}\right),\left(B_{1}, B_{2}\right)$ be quasi-Banach couples and let $\left(E_{1}, E_{2}\right)$ be an $r$-Banach couple, $0<r \leq 1$. Let $0<\theta<1$ and $0<q_{1}, q_{2}, q \leq \infty$ with

$$
\frac{1}{q}= \begin{cases}\frac{1}{q_{1}}+\frac{1}{q_{2}}-\frac{1}{r} & \text { if } q_{1}, q_{2} \geq r \\ \frac{1}{\max \left(q_{1}, q_{2}\right)} & \text { if } q_{1}<r \text { or } q_{2}<r .\end{cases}
$$

If $T$ is a bilinear operator defined on $\left(A_{1} \cap A_{2}\right) \times\left(B_{1} \cap B_{2}\right)$ with values in $E_{1} \cap E_{2}$ such that

$$
\left\|T(a, b)\left|E_{j}\left\|\leq M_{j}\right\| a\right| A_{j}\right\|\left\|b \mid B_{j}\right\|, \quad a \in A_{1} \cap A_{2}, b \in B_{1} \cap B_{2}, j=1,2
$$

then

$$
\left\|T(a, b)\left|\left(E_{1}, E_{2}\right)_{\theta, q}\left\|\leq C M_{1}^{1-\theta} M_{2}^{\theta}\right\| a\right|\left(A_{1}, A_{2}\right)_{\theta, q_{1}}\right\|\left\|b \mid\left(B_{1}, B_{2}\right)_{\theta, q_{2}}\right\|,
$$

$a \in A_{1} \cap A_{2}$ and $b \in B_{1} \cap B_{2}$. Furthermore, if $q_{j}<\infty$ for $j=1,2$, then $T$ may be uniquely extended to a bounded bilinear operator from $\left(A_{1}, A_{2}\right)_{\theta, q_{1}} \times\left(B_{1}, B_{2}\right)_{\theta, q_{2}}$ to $\left(E_{1}, E_{2}\right)_{\theta, q}$.

It has been shown by Janson [27, Theorem 2] that under extra assumptions on the operator $T$ then the value of the parameter $q$ can be improved with the result that $\frac{1}{q}=\frac{1}{q_{1}}+\frac{1}{q_{2}}$.

If $A$ is a quasi-Banach space and $\lambda>0$, we write $\lambda A$ for the space $A$ quasinormed by $\lambda\|a \mid A\|$. It is not hard to check that if $\left(A_{1}, A_{2}\right)$ is a quasi-Banach couple and $\lambda_{1}, \lambda_{2}>0$ then

$$
\left(\lambda_{1} A_{1}, \lambda_{2} A_{2}\right)_{\theta, q}=\lambda_{1}^{1-\theta} \lambda_{2}^{\theta}\left(A_{1}, A_{2}\right)_{\theta, q}
$$

The following examples of real interpolation spaces will be important in our later considerations. 
Put $\mathbb{N}_{0}=\mathbb{N} \cup\{0\}$. Let $s \in \mathbb{R}, 0<q \leq \infty$ and let $A$ be a quasi-Banach space. We denote by $\ell_{q}^{s}(A)$ the space of all sequences $\left(a_{k}\right)_{k \in \mathbb{N}_{0}} \subseteq A$ such that

$$
\left\|\left(a_{k}\right) \mid \ell_{q}^{s}(A)\right\|=\left(\sum_{k=0}^{\infty} 2^{k s q}\left\|a_{k} \mid A\right\|^{q}\right)^{1 / q}<\infty
$$

(with the usual modification if $q=\infty$ ).

For $0<q_{1}, q_{2}, q \leq \infty,-\infty<s_{1} \neq s_{2}<\infty, 0<\theta<1$ and $s=(1-\theta) s_{1}+\theta s_{2}$ we have, with equivalent quasi-norms,

$$
\left(\ell_{q_{1}}^{s_{1}}(A), \ell_{q_{2}}^{s_{2}}(A)\right)_{\theta, q}=\ell_{q}^{s}(A) .
$$

Equality (2.2) is a consequence of [47, Theorem 1.18.2] and [6, Theorem 5.6.1] extended to quasi-Banach spaces $A$.

When $A=\mathbb{C}$ we simply write $\ell_{q}^{s}$. Next we use spaces $\ell_{q}^{s}$ to show that the result of Lemma 2.1 is the best possible.

Example 2.3 Let $0<q<p \leq 1,-\infty<s_{1} \neq s_{2}<\infty, 0<\theta<1$ and put $s=(1-\theta) s_{1}+\theta s_{2}$. Consider the $p$-Banach couple $\left(\ell_{p}^{s_{1}}, \ell_{p}^{s_{2}}\right)$. Formula (2.2) implies that

$$
\left(\ell_{p}^{s_{1}}, \ell_{p}^{s_{2}}\right)_{\theta, q}=\ell_{q}^{s},
$$

and proceeding as we did with $\ell_{q}$ but using now the sequence $v_{j}=2^{-j s} e_{j}$, it turns out that $\ell_{q}^{s}$ is not $\rho$-Banach if $\rho>q=\min (p, q)$.

Let $\left(\lambda_{k}\right)_{k \in \mathbb{N}_{0}}$ be a sequence of positive numbers and let $\left(W_{k}\right)_{k \in \mathbb{N}_{0}}$ be a sequence of quasi-Banach spaces with the same constant $c \geq 1$ in the quasi-triangle inequality for any $W_{k}$. For $0<q \leq \infty$, we designate by $\ell_{q}\left(\lambda_{k} W_{k}\right)$ the collection of all those sequences $w=\left(w_{k}\right)_{k \in \mathbb{N}_{0}}$ such that $w_{k} \in W_{k}$ and the quasi-norm

$$
\left\|w \mid \ell_{q}\left(\lambda_{k} W_{k}\right)\right\|=\left(\sum_{k=0}^{\infty} \lambda_{k}^{q}\left\|w_{k} \mid W_{k}\right\|^{q}\right)^{1 / q}
$$

is finite (with the usual modification if $q=\infty$ ).

Let $\left(A_{k}, B_{k}\right)$ be interpolation couples of quasi-Banach spaces with the same constant in the quasi-triangle inequality. Let $\left(\lambda_{k}\right)_{k \in \mathbb{N}_{0}},\left(\mu_{k}\right)_{k \in \mathbb{N}_{0}}$ be sequences of positive numbers, $0<p_{1}, p_{2}<\infty, 0<\theta<1$ and $\frac{1}{p}=\frac{1-\theta}{p_{1}}+\frac{\theta}{p_{2}}$. Then we have

$$
\left(\ell_{p_{1}}\left(\lambda_{k} A_{k}\right), \ell_{p_{2}}\left(\mu_{k} B_{k}\right)\right)_{\theta, p}=\ell_{p}\left(\left(\lambda_{k} A_{k}, \mu_{k} B_{k}\right)_{\theta, p}\right)=\ell_{p}\left(\lambda_{k}^{1-\theta} \mu_{k}^{\theta}\left(A_{k}, B_{k}\right)_{\theta, p}\right)
$$

(see [47, Theorem 1.18.1, Remark 1.18.1/4, pp. 120-123]).

Next fix $n \in \mathbb{N}$ and consider the Euclidean $n$-space $\mathbb{R}^{n}$ endowed with the Lebesgue measure. Let $A$ be a quasi-Banach space and let $0<p \leq \infty$. We put $L_{p}(A)=$ $L_{p}\left(\mathbb{R}^{n} ; A\right)$ for the usual vector-valued $L_{p}$-space in the sense of the Bochner integral. If $A=\mathbb{C}$, we write $L_{p}\left(\mathbb{R}^{n}\right)$.

Given $0<p<\infty$ and $0<r \leq \infty$, the Lorentz space $L_{p, r}(A)=L_{p, r}\left(\mathbb{R}^{n} ; A\right)$ consists of all (equivalence classes of) strongly measurable functions with values in $A$ which have a finite quasi-norm

$$
\left\|f \mid L_{p, r}(A)\right\|=\left(\int_{0}^{\infty}\left(t^{1 / p} f^{*}(t)\right)^{r} \frac{d t}{t}\right)^{1 / r}
$$


(the integral should be replaced by the supremum if $r=\infty$ ). Here $f^{*}$ is the nonincreasing rearrangement of $f$

$$
f^{*}(t)=\inf \left\{s>0:\left|\left\{x \in \mathbb{R}^{n}:\|f(x) \mid A\|>s\right\}\right| \leq t\right\},
$$

where $|\Gamma|$ stands for the Lebesgue measure of the measurable set $\Gamma$.

Note that if $p=r$, then $L_{p, p}(A)=L_{p}(A)$. If $A=\mathbb{C}$, we simply write $L_{p, r}\left(\mathbb{R}^{n}\right)$. See $[34,47,6]$. See also $[26,42,5,17]$ for Lorentz spaces in the scalar case. When $0<r<q \leq \infty$ we have that $L_{p, r}\left(\mathbb{R}^{n}\right) \hookrightarrow L_{p, q}\left(\mathbb{R}^{n}\right)$.

Let $0<p<\infty$. For the couple $\left(L_{p}(A), L_{\infty}(A)\right)$, the $K$-functional satisfies that

$$
K\left(t, f ; L_{p}(A), L_{\infty}(A)\right) \sim\left(\int_{0}^{t^{p}}\left(f^{*}(s)\right)^{p} d s\right)^{1 / p}
$$

(see [34]). This yields that for $0<\theta<1$ and $0<r \leq \infty$, we have with equivalent quasi-norms

$$
\left(L_{p}(A), L_{\infty}(A)\right)_{\theta, r}=L_{u, r}(A), \frac{1}{u}=\frac{1-\theta}{p} .
$$

Using the reiteration formula for the real method, we derive with equivalent quasinorms

$$
\left(L_{p_{1}, r_{1}}(A), L_{p_{2}, r_{2}}(A)\right)_{\theta, r}=L_{p, r}(A)
$$

where $0<r_{1}, r_{2}, r \leq \infty, 0<p_{1} \neq p_{2} \leq \infty, 0<\theta<1$ and $\frac{1}{p}=\frac{1-\theta}{p_{1}}+\frac{\theta}{p_{2}}$ (see [47, (16), p. 134 and Remark 5, p. 135] or [6, Sections 5.2 and 5.3, pp. 108-114]).

Consider now the scalar Lorentz function spaces. The space $L_{p, r}\left(\mathbb{R}^{n}\right)$ is a Banach space (with an equivalent norm) if, and only if, either $1<p<\infty, 1 \leq r \leq \infty$, or $p=r=1$ (see [26, (2.6), p. 259]). The remaining spaces $L_{p, r}\left(\mathbb{R}^{n}\right)$ have an equivalent $\rho$-norm for any $0<\rho<p$ and $\rho \leq \min (r, 1)$ (see [42, Theorem 18] or use Lemma 2.1 and (2.4)). This property can be improved in some cases reaching the value $p$. Namely, if $0<p<1$ and $p<r \leq \infty$ then $L_{p, r}\left(\mathbb{R}^{n}\right)$ has an equivalent $p$-norm. This result for $r=\infty$ was proved by Kalton [29, Theorem 6.1] and in the case $p<r<\infty$ by Seeger and Trebels [43, Proposition B.1].

Next we consider some properties of the multiplication of functions. By $X \cdot Y \hookrightarrow Z$ we mean that there is a constant $c>0$ such that $\|f g|Z\|\leq c\| f| X\| \cdot\|g \mid Y\|$, for any $f \in X$ and $g \in Y$.

Classical Hölder inequality says that

$$
L_{p_{1}}\left(\mathbb{R}^{n}\right) \cdot L_{p_{2}}\left(\mathbb{R}^{n}\right) \hookrightarrow L_{p}\left(\mathbb{R}^{n}\right)
$$

provided that $0<p_{1}, p_{2} \leq \infty$ and $\frac{1}{p}=\frac{1}{p_{1}}+\frac{1}{p_{2}}$.

For Lorentz spaces, the corresponding result reads

$$
L_{p_{1}, q_{1}}\left(\mathbb{R}^{n}\right) \cdot L_{p_{2}, q_{2}}\left(\mathbb{R}^{n}\right) \hookrightarrow L_{p, q}\left(\mathbb{R}^{n}\right)
$$

provided that $0<p_{1}, p_{2}<\infty, \frac{1}{p}=\frac{1}{p_{1}}+\frac{1}{p_{2}}, 0<q_{1}, q_{2} \leq \infty$ and $\frac{1}{q}=\frac{1}{q_{1}}+\frac{1}{q_{2}}$. Next we establish it by using bilinear interpolation. 
Take any $0<r<p$. The bilinear operator $T(f, g)=f g$ is clearly bounded in the following three cases

$$
\left\{\begin{array}{l}
T: L_{\infty}\left(\mathbb{R}^{n}\right) \times L_{\infty}\left(\mathbb{R}^{n}\right) \longrightarrow L_{\infty}\left(\mathbb{R}^{n}\right), \\
T: L_{r}\left(\mathbb{R}^{n}\right) \times L_{\infty}\left(\mathbb{R}^{n}\right) \longrightarrow L_{r}\left(\mathbb{R}^{n}\right), \\
T: L_{\infty}\left(\mathbb{R}^{n}\right) \times L_{r}\left(\mathbb{R}^{n}\right) \longrightarrow L_{r}\left(\mathbb{R}^{n}\right) .
\end{array}\right.
$$

Take $\left(A_{1}, A_{2}\right)=\left(B_{1}, B_{2}\right)=\left(E_{1}, E_{2}\right)=\left(L_{\infty}\left(\mathbb{R}^{n}\right), L_{r}\left(\mathbb{R}^{n}\right)\right)$. For $\theta_{j}=r / p_{j}, j=1,2$, we have that $\left(L_{\infty}\left(\mathbb{R}^{n}\right), L_{r}\left(\mathbb{R}^{n}\right)\right)_{\theta_{j}, q_{j}}=L_{p_{j}, q_{j}}\left(\mathbb{R}^{n}\right)$. Then, (2.7) follows by applying the bilinear interpolation result of Janson [27, Theorem 2] with $\alpha_{0}=0, \alpha_{1}=\alpha_{2}=1$ and $\theta=\alpha+\alpha_{1} \theta_{1}+\alpha_{2} \theta_{2}=\theta_{1}+\theta_{2}=\frac{r}{p}$.

Janson's theorem also allows us to establish the following result on convolution of Lorentz spaces that we shall need later.

Let $1<p_{1}, p_{2}<\infty, \frac{1}{p}=\frac{1}{p_{1}}+\frac{1}{p_{2}}-1>0,0<q_{1}, q_{2} \leq \infty$ and $\frac{1}{q} \leq \frac{1}{q_{1}}+\frac{1}{q_{2}}$. Then the convolution operator satisfies that

$$
*: L_{p_{1}, q_{1}}\left(\mathbb{R}^{n}\right) \times L_{p_{2}, q_{2}}\left(\mathbb{R}^{n}\right) \longrightarrow L_{p, q}\left(\mathbb{R}^{n}\right) \text { boundedly }
$$

For the proof one can apply [27, Theorem 2] with

$$
\left(A_{1}, A_{2}\right)=\left(B_{1}, B_{2}\right)=\left(E_{1}, E_{2}\right)=\left(L_{\infty}\left(\mathbb{R}^{n}\right), L_{1}\left(\mathbb{R}^{n}\right)\right), \alpha_{0}=-1 \text { and } \alpha_{1}=\alpha_{2}=1 .
$$

See also the paper by O'Neil [36, Theorem 2.6].

\section{Triebel-Lizorkin-Lorentz spaces}

Let $\mathcal{S}\left(\mathbb{R}^{n}\right)$ be the Schwartz space of all complex-valued rapidly decreasing infinitely differentiable functions on $\mathbb{R}^{n}$. We write $\mathcal{S}^{\prime}\left(\mathbb{R}^{n}\right)$ for the space of all tempered distributions. For $f \in \mathcal{S}^{\prime}\left(\mathbb{R}^{n}\right)$, we put $\hat{f}$ for its Fourier transform and $\check{f}$ for its inverse Fourier transform.

Let $\varphi_{0} \in \mathcal{S}\left(\mathbb{R}^{n}\right)$ such that

$$
\varphi_{0}(x)=1 \text { if }|x| \leq 1 \text { and } \varphi_{0}(x)=0 \text { if }|x| \geq 3 / 2 .
$$

Here $|x|$ stands for the norm in $\mathbb{R}^{n}$. For $k \in \mathbb{N}$ put $\varphi_{k}(x)=\varphi_{0}\left(2^{-k} x\right)-\varphi_{0}\left(2^{-k+1} x\right)$ Since

$$
\sum_{k=0}^{\infty} \varphi_{k}(x)=1 \text { for all } x \in \mathbb{R}^{n},
$$

the sequence $\left(\varphi_{k}\right)_{k \in \mathbb{N}_{0}}$ is a smooth dyadic resolution of unity.

Definition 3.1 Let $s \in \mathbb{R}, 0<p<\infty$ and $0<q, r \leq \infty$. The Triebel-Lizorkin-Lorentz space $F_{q}^{s} L_{p, r}\left(\mathbb{R}^{n}\right)$ is formed by all $f \in \mathcal{S}^{\prime}\left(\mathbb{R}^{n}\right)$ having a finite quasi-norm

$$
\left\|f\left|F_{q}^{s} L_{p, r}\left(\mathbb{R}^{n}\right)\|=\|\left(\sum_{j=0}^{\infty}\left[2^{j s}\left|\left(\varphi_{j} \hat{f}\right)^{\vee}(\cdot)\right|\right]^{q}\right)^{1 / q}\right| L_{p, r}\left(\mathbb{R}^{n}\right)\right\| .
$$


Definition 3.1 extends [47, Definition 2.4.2, p.184] to the full range of parameters. Note that in [47], Triebel-Lizorkin-Lorentz spaces are denoted by $F_{p, q,(r)}^{s}\left(\mathbb{R}^{n}\right)$. Other notations used for these spaces are $F_{p, q}^{s, r}$ (in [25]), $F_{q}^{s}\left[L^{p, r}\right]$ (in [43]) and $L_{s, q}^{p, r}$ ([59]). Clearly, when $r=p$, then we recover the usual Triebel-Lizorkin spaces $F_{q}^{s} L_{p, p}\left(\mathbb{R}^{n}\right)=$ $F_{p, q}^{s}\left(\mathbb{R}^{n}\right)$.

Spaces $F_{q}^{s} L_{p, r}\left(\mathbb{R}^{n}\right)$ are quasi-Banach spaces. They are Banach spaces if $p>1$ and $\min (q, r) \geq 1$. For the Banach case, it is shown in [47, Remark 1, p. 184] that spaces $F_{q}^{s} L_{p, r}\left(\mathbb{R}^{n}\right)$ are independent of the generating function $\varphi_{0}$ (with equivalent norms). For the general case the independence of $\varphi_{0}$ is established in [59, Theorem 2].

We have with equivalence of quasi-norms

$$
L_{p, r}\left(\mathbb{R}^{n}\right)=F_{2}^{0} L_{p, r}\left(\mathbb{R}^{n}\right) \text { provided } 1<p<\infty \text { and } 0<r \leq \infty .
$$

This assertion of Littlerwood-Paley type for Lorentz spaces has been proved by Yang, Cheng and Peng [59, Theorem 5] by using wavelets. It can also be derived proceeding as in the case of $L_{p}$ (see [48, Theorem 2.5.6/Step 2]) and using the corresponding Lorentz version of the Hörmander's multiplier theorem for $\ell_{2}$ [48, Proposition 2.5.6].

Wavelets will be important for the rest of this section. Next we review some notation and basic results. We follow mainly [50,51].

For $u \in \mathbb{N}$, let $C^{u}(\mathbb{R})$ be the space of all complex-valued continuous functions on $\mathbb{R}$ having continuous bounded derivatives up to order $u$ inclusively. Let

$$
\psi_{F} \in C^{u}(\mathbb{R}), \quad \psi_{M} \in C^{u}(\mathbb{R})
$$

be real compactly supported Daubechies wavelets with

$$
\int_{\mathbb{R}} \psi_{M}(x) x^{v} d x=0 \text { for all } v \in \mathbb{N}_{0} \text { with } v<u .
$$

We also assume that $\psi_{F}, \psi_{M}$ have $L_{2}$-norm 1 . Let $G=\left(G_{1}, \ldots, G_{n}\right) \in G^{0}=\{F, M\}^{n}$, which means that each $G_{r}$ is either $F$ or $M$. For $j \in \mathbb{N}$, we define $G^{j}=\{F, M\}^{n *}$ as the collection of all $G=\left(G_{1}, \ldots, G_{n}\right)$ such that each $G_{r}$ is either $F$ or $M$ but at least one of the $G_{r}$ must be $M$. Put

$$
\psi_{G, m}^{j}(x)=2^{j n / 2} \prod_{r=1}^{n} \psi_{G_{r}}\left(2^{j} x_{r}-m_{r}\right), G \in G^{j}, m \in \mathbb{Z}^{n},
$$

where now $j \in \mathbb{N}_{0}$. Then

$$
\left\{\psi_{G, m}^{j}: j \in \mathbb{N}_{0}, G \in G^{j}, m \in \mathbb{Z}^{n}\right\}
$$

is an orthonormal basis in $L_{2}\left(\mathbb{R}^{n}\right)$ and

$$
f=\sum_{j, G, m} \lambda_{m}^{j, G} 2^{-j n / 2} \psi_{G, m}^{j}
$$

with

$$
\lambda_{m}^{j, G}=\lambda_{m}^{j, G}(f)=2^{j n / 2} \int_{\mathbb{R}^{n}} f(x) \psi_{G, m}^{j}(x) d x=2^{j n / 2}\left(f, \psi_{G, m}^{j}\right)
$$


where $2^{-j n / 2} \psi_{G, m}^{j}$ are uniformly bounded functions (with respect to $j$ and $m$ ). See [51, Section 1.2.1].

Let $\chi_{j, m}$ be the characteristic function of the dyadic cube $Q_{j, m}=2^{-j} m+2^{-j}(0,1)^{n}$ in $\mathbb{R}^{n}$ with sides of length $2^{-j}$ parallel to the axes of coordinates and $2^{-j} m$ as the lower left corner.

Let $s \in \mathbb{R}, 0<p<\infty$ and $0<q \leq \infty$. The space $f_{p, q}^{s}$ consists of all sequences

$$
\lambda=\left\{\lambda_{m}^{j, G} \in \mathbb{C}: j \in \mathbb{N}_{0}, G \in G^{j}, m \in \mathbb{Z}^{n}\right\}
$$

such that

$$
\left\|\lambda\left|f_{p, q}^{s}\|=\|\left(\sum_{j, G, m} 2^{j s q}\left|\lambda_{m}^{j, G} \chi_{j, m}(\cdot)\right|^{q}\right)^{1 / q}\right| L_{p}\left(\mathbb{R}^{n}\right)\right\|<\infty
$$

with the usual modifications if $q=\infty$.

Put

$$
I(f)=\left(\lambda_{m}^{j, G}\right)=\left(2^{j n / 2}\left(f, \psi_{G, m}^{j}\right)\right) .
$$

As one can see in [51, Section 1.2.2], if we choose $u$ sufficiently large, then we have for Triebel-Lizorkin spaces that $f \in \mathcal{S}^{\prime}\left(\mathbb{R}^{n}\right)$ belongs to $F_{p, q}^{s}\left(\mathbb{R}^{n}\right)$ if, and only if, $\lambda \in f_{p, q}^{s}$. Moreover, the map $I$ in (3.4) is an isomorphic map of $F_{p, q}^{s}\left(\mathbb{R}^{n}\right)$ onto $f_{p, q}^{s}$.

Next we introduce the sequence spaces which are relevant to deal with TriebelLizorkin-Lorentz spaces.

Definition 3.2 Let $s \in \mathbb{R}, 0<p<\infty$ and $0<q, r \leq \infty$.

(a) The space $f_{q}^{s} L_{p, r}$ is the collection of all sequences $\lambda=\left(\lambda_{m}^{j, G}\right)$ in (3.3) having a finite quasi-norm

$$
\left\|\lambda\left|f_{q}^{s} L_{p, r}\|=\|\left(\sum_{j, G, m} 2^{j s q}\left|\lambda_{m}^{j, G} \chi_{j, m}(\cdot)\right|^{q}\right)^{1 / q}\right| L_{p, r}\left(\mathbb{R}^{n}\right)\right\| .
$$

(b) The space $\mathbf{L}_{p, r}\left(\ell_{q}^{s}\right)$ consists of all vector-valued functions

$$
g=\left\{g_{m}^{j, G}: j \in \mathbb{N}_{0}, G \in G^{j}, m \in \mathbb{Z}^{n}\right\}
$$

such that the quasi-norm

$$
\left\|g\left|\mathbf{L}_{p, r}\left(\ell_{q}^{s}\right)\|=\|\left(\sum_{j, G, m} 2^{j s q}\left|g_{m}^{j, G}(\cdot)\right|^{q}\right)^{1 / q}\right| L_{p, r}\left(\mathbb{R}^{n}\right)\right\|
$$

is finite. If $p=r$, we designate this space by $\mathbf{L}_{p}\left(\ell_{q}^{s}\right)$.

Note that $f_{q}^{s} L_{p, p}=f_{p, q}^{s}$.

The following characterization of Triebel-Lizorkin-Lorentz spaces in terms of wavelets is due to Yang, Cheng and Peng [59, Theorems 3 and 4]. 
Theorem 3.3 Let $0<p<\infty, 0<q, r \leq \infty, s \in \mathbb{R}$ and let $\psi_{G, m}^{j}$ be the wavelets (3.2) with $u$ being sufficiently large. Let $f \in \mathcal{S}^{\prime}\left(\mathbb{R}^{n}\right)$. Then $f \in F_{q}^{s} L_{p, r}\left(\mathbb{R}^{n}\right)$ if, and only if, $f$ can be represented as

$$
f=\sum_{j, G, m} \lambda_{m}^{j, G} 2^{-j n / 2} \psi_{G, m}^{j} \text { with } \lambda \in f_{q}^{s} L_{p, r}
$$

(summability in $\mathcal{S}^{\prime}\left(\mathbb{R}^{n}\right)$ ). The representation (3.6) is unique with

$$
\lambda_{m}^{j, G}=2^{j n / 2}\left(f, \psi_{G, m}^{j}\right)
$$

and the map I in (3.4) is an isomorphism from $F_{q}^{s} L_{p, r}\left(\mathbb{R}^{n}\right)$ onto $f_{q}^{s} L_{p, r}$.

Remark 3.4 As a consequence of Theorems 3.3 and (3.1), we obtain a characterization for $L_{p, r}\left(\mathbb{R}^{n}\right)$ in terms of wavelets when $1<p<\infty$ and $0<r \leq \infty$. This problem in the case of spaces on domains and $1 \leq r \leq \infty$ has been studied by Triebel in [52] and [51, Section 2.5.2].

The characterization of $F_{q}^{s} L_{p, r}\left(\mathbb{R}^{n}\right)$ by real interpolation was established by Triebel [47, Theorem 2.4.2/1, (c), p. 185] in the Banach case by using the so-called retraction and co-retraction method. Consider a smooth dyadic resolution of unity $\left(\varphi_{k}\right)_{k=0}^{\infty}$ and for any $f \in \mathcal{S}^{\prime}\left(\mathbb{R}^{n}\right)$, put $J(f)=\left(\left(\varphi_{k} \hat{f}\right)^{\vee}\right)$. Moreover, for any $\left(f_{k}\right) \in L_{p}\left(\ell_{q}^{s}\right)$, define $R\left(f_{k}\right)=\sum_{k=0}^{\infty}\left(\tilde{\varphi}_{k} \hat{f}_{k}\right)^{\vee}$, where $\tilde{\varphi}_{k}=\varphi_{k-1}+\varphi_{k}+\varphi_{k+1}$ and $\varphi_{-1} \equiv 0$. It turns out that

$$
J: F_{p, q}^{s}\left(\mathbb{R}^{n}\right) \longrightarrow L_{p}\left(\ell_{q}^{s}\right) \quad \text { and } \quad R: L_{p}\left(\ell_{q}^{s}\right) \longrightarrow F_{p, q}^{s}\left(\mathbb{R}^{n}\right)
$$

are bounded linear operators with $R(J f)=f$. Then it follows from [47, Theorem 1.2.4] and (2.5) that

$$
\left(F_{p_{1}, q}^{s}\left(\mathbb{R}^{n}\right), F_{p_{2}, q}^{s}\left(\mathbb{R}^{n}\right)\right)_{\theta, r}=F_{q}^{s} L_{p, r}\left(\mathbb{R}^{n}\right)
$$

provided that $-\infty<s<\infty, 1 \leq p_{1} \neq p_{2}<\infty, 1 \leq q, r \leq \infty, 0<\theta<1$ and $\frac{1}{p}=\frac{1-\theta}{p_{1}}+\frac{\theta}{p_{2}}$. But the operator $R$ is meaningless for $0<p<1$. A new approach was given by Yang, Cheng and Peng [59, Theorem 6] based on the wavelet characterization of $F_{q}^{s} L_{p, r}\left(\mathbb{R}^{n}\right)$ and the computation of the $K$-functional with the help of wavelets. As a result, they characterized $F_{q}^{s} L_{p, r}\left(\mathbb{R}^{n}\right)$ by real interpolation for the whole range of parameters. Since that interpolation result is the key point for the rest of our results, next we show another proof for it based on spaces $f_{p, q}^{s}$ and Theorem 3.3.

Note that spaces $f_{p, q}^{s}$ and $f_{q}^{s} L_{p, r}$ can be identified with subspaces of $\mathbf{L}_{p}\left(\ell_{q}^{s}\right)$ and $\mathbf{L}_{p, r}\left(\ell_{q}^{s}\right)$, respectively. Moreover, applying (2.5), we get

$$
\left(\mathbf{L}_{p_{1}}\left(\ell_{q}^{s}\right), \mathbf{L}_{p_{2}}\left(\ell_{q}^{s}\right)\right)_{\theta, r}=\mathbf{L}_{p, r}\left(\ell_{q}^{s}\right)
$$

provided that $-\infty<s<\infty, 0<q \leq \infty, 0<p_{1}<p_{2}<\infty, 0<\theta<1$ and $\frac{1}{p}=\frac{1-\theta}{p_{1}}+\frac{\theta}{p_{2}}$.

We are going to characterize $f_{q}^{s} L_{p, r}$ by interpolation with the help of (3.7) and well-known vector-valued maximal inequalities. 
Let

$$
(M f)(x)=\sup _{x \in Q}|Q|^{-1} \int_{Q}|f(y)| d y
$$

be the Hardy-Littlewood maximal function. Let $0<p<\infty, 0<q \leq \infty$ and $0<w<\min (p, q)$. Then, according to the Fefferman-Stein maximal inequality (see [46, Theorem XII.1.1, p. 303] or [23, Theorem 4.6.6, p. 335]) we have

$$
\left\|\left(\sum_{j, G, m} 2^{j s q}\left(M\left|g_{m}^{j, G}\right|^{w}(\cdot)\right)^{q / w}\right)^{1 / q}\left|L_{p}\left(\mathbb{R}^{n}\right)\|\leq c\|\left(g_{m}^{j, G}\right)\right| \mathbf{L}_{p}\left(\ell_{q}^{s}\right)\right\| .
$$

Theorem 3.5 Let $s \in \mathbb{R}, 0<q, r \leq \infty, 0<p_{1}<p_{2}<\infty, 0<\theta<1$ and $\frac{1}{p}=\frac{1-\theta}{p_{1}}+\frac{\theta}{p_{2}}$. Then we have, with equivalent quasi-norms

$$
\left(f_{p_{1}, q}^{s}, f_{p_{2}, q}^{s}\right)_{\theta, r}=f_{q}^{s} L_{p, r}
$$

Proof For $\lambda=\left(\lambda_{m}^{j, G}\right)_{j \in \mathbb{N}_{0}, G \in G^{j}, m \in \mathbb{Z}^{n}} \in f_{p_{k}, q}^{s}, k=1,2$, put

$$
R(\lambda)(x)=\left(\lambda_{m}^{j, G} \chi_{j, m}(x)\right)_{j, G, m} .
$$

It is clear that $R: f_{p_{k}, q}^{s} \longrightarrow \mathbf{L}_{p_{k}}\left(\ell_{q}^{s}\right)$ is bounded with $\left\|R(\lambda)\left|\mathbf{L}_{p_{k}}\left(\ell_{q}^{s}\right)\|=\| \lambda\right| f_{p_{k}, q}^{s}\right\|$, $k=1,2$.

Take any $0<w<\min \left(p_{1}, q\right)$ and consider the operator $P$ which assigns to any $g=\left(g_{m}^{j, G}\right)_{j, G, m}$ the sequence

$$
P\left(g_{m}^{j, G}\right)=\left(\left(\frac{1}{\left|Q_{j, m}\right|} \int_{Q_{j, m}}\left|g_{m}^{j, G}(y)\right|^{w} d y\right)^{1 / w}\right)_{j, G, m} .
$$

Applying (3.8) we derive

$$
\begin{aligned}
& \left\|P\left(g_{m}^{j, G}\right) \mid f_{p_{k}, q}^{s}\right\| \\
& \quad=\left\|\left(\sum_{j, G, m}\left[2^{j s}\left(\frac{1}{\left|Q_{j, m}\right|} \int_{Q_{j, m}}\left|g_{m}^{j, G}(y)\right|^{w} d y\right)^{1 / w} \chi_{j, m}(\cdot)\right]^{q}\right)^{w / q} \mid L_{p_{k} / w}\left(\mathbb{R}^{n}\right)\right\|^{1 / w} \\
& \quad \leq\left\|\left(\sum_{j, G, m}\left[2^{j s w} M\left|g_{m}^{j, G}\right|^{w}(\cdot)\right]^{q / w}\right)^{w / q} \mid L_{p_{k} / w}\left(\mathbb{R}^{n}\right)\right\|^{1 / w} \\
& \quad \leq c\left\|\left(g_{m}^{j, G}\right) \mid \mathbf{L}_{p_{k}}\left(\ell_{q}^{s}\right)\right\| .
\end{aligned}
$$

Whence, $P: \mathbf{L}_{p_{k}}\left(\ell_{q}^{s}\right) \longrightarrow f_{p_{k}, q}^{s}$ is bounded. Note also that

$$
(P \circ R)(\lambda)=\left(\left|\lambda_{m}^{j, G}\right|\right)_{j, G, m}=|\lambda| .
$$

The linearity and boundedness of $R$ imply that for every $\lambda \in f_{p_{1}, q}^{s}+f_{p_{2}, q}^{s}$ we get

$$
K\left(t, R(\lambda) ; \mathbf{L}_{p_{1}}\left(\ell_{q}^{s}\right), \mathbf{L}_{p_{2}}\left(\ell_{q}^{s}\right)\right) \leq K\left(t, \lambda ; f_{p_{1}, q}^{s}, f_{p_{2}, q}^{s}\right) .
$$

On the other hand, due to the lattice properties of $f_{p_{k}, q}^{s}$, the last $K$-functional is

$$
\begin{aligned}
& K\left(t, \lambda ; f_{p_{1}, q}^{s}, f_{p_{2}, q}^{s}\right) \\
& \quad=\inf \left\{\left\|\left(\lambda_{m, 1}^{j, G}\right)\left|f_{p_{1}, q}^{s}\|+t\|\left(\lambda_{m, 2}^{j, G}\right)\right| f_{p_{2}, q}^{s}\right\|:\left|\lambda_{m}^{j, G}\right| \leq \lambda_{m, 1}^{j, G}+\lambda_{m, 2}^{j, G}, \lambda_{m, 1}^{j, G}, \lambda_{m, 2}^{j, G} \geq 0\right\}
\end{aligned}
$$


(see, for example, [15, Lemma 3.1]). This together with the fact that $|P f| \leq c_{w}\left(P\left|f_{1}\right|+\right.$ $\left.P\left|f_{2}\right|\right)$ if $f=f_{1}+f_{2}$, imply that

$$
\begin{aligned}
K\left(t, P f ; f_{p_{1}, q}^{s}, f_{p_{2}, q}^{s}\right) & =K\left(t,|P f| ; f_{p_{1}, q}^{s}, f_{p_{2}, q}^{s}\right) \\
& \leq c_{w} K\left(t, P\left|f_{1}\right|+P\left|f_{2}\right| ; f_{p_{1}, q}^{s}, f_{p_{2}, q}^{s}\right) \\
& \lesssim\left\|P \left|f_{1}\left\|f_{p_{1}, q}^{s}\right\|+t\left\|P \mid f_{2}\right\| f_{p_{2}, q}^{s} \|\right.\right. \\
& \lesssim\left\|f_{1}\left|\mathbf{L}_{p_{1}}\left(\ell_{q}^{s}\right)\|+t\| f_{2}\right| \mathbf{L}_{p_{2}}\left(\ell_{q}^{s}\right)\right\| .
\end{aligned}
$$

Taking the infimum over all possible decompositions we obtain that

$$
K\left(t, P f ; f_{p_{1}, q}^{s}, f_{p_{2}, q}^{s}\right) \lesssim K\left(t, f ; \mathbf{L}_{p_{1}}\left(\ell_{q}^{s}\right), \mathbf{L}_{p_{2}}\left(\ell_{q}^{s}\right)\right)
$$

for every $f \in \mathbf{L}_{p_{1}}\left(\ell_{q}^{s}\right)+\mathbf{L}_{p_{2}}\left(\ell_{q}^{s}\right)$. Thus,

$$
\begin{aligned}
K\left(t, \lambda ; f_{p_{1}, q}^{s}, f_{p_{2}, q}^{s}\right) & =K\left(t,|\lambda| ; f_{p_{1}, q}^{s}, f_{p_{2}, q}^{s}\right) \\
& =K\left(t,(P \circ R)(\lambda) ; f_{p_{1}, q}^{s}, f_{p_{2}, q}^{s}\right) \\
& \lesssim K\left(t, R(\lambda) ; \mathbf{L}_{p_{1}}\left(\ell_{q}^{s}\right), \mathbf{L}_{p_{2}}\left(\ell_{q}^{s}\right)\right) .
\end{aligned}
$$

From (3.9), (3.10) and (3.7), we conclude that

$$
\begin{aligned}
\left\|\lambda \mid\left(f_{p_{1}, q}^{s}, f_{p_{2}, q}^{s}\right)_{\theta, r}\right\| & \sim\left\|R \lambda \mid\left(\mathbf{L}_{p_{1}}\left(\ell_{q}^{s}\right), \mathbf{L}_{p_{2}}\left(\ell_{q}^{s}\right)\right)_{\theta, r}\right\| \\
& \sim\left\|R \lambda\left|\mathbf{L}_{p, r}\left(\ell_{q}^{s}\right)\|=\| \lambda\right| f_{q}^{s} L_{p, r}\right\| .
\end{aligned}
$$

Theorem 3.6 Let $s \in \mathbb{R}$ and $0<q, r_{1}, r_{2}, r \leq \infty$. Assume that $0<\theta<1,0<p_{1} \neq$ $p_{2}<\infty$ and $\frac{1}{p}=\frac{1-\theta}{p_{1}}+\frac{\theta}{p_{2}}$. Then we have with equivalent quasi-norms

(a) $\left(F_{p_{1}, q}^{s}\left(\mathbb{R}^{n}\right), F_{p_{2}, q}^{s}\left(\mathbb{R}^{n}\right)\right)_{\theta, r}=F_{q}^{s} L_{p, r}\left(\mathbb{R}^{n}\right)$.

(b) $\left(F_{q}^{s} L_{p_{1}, r_{1}}\left(\mathbb{R}^{n}\right), F_{q}^{s} L_{p_{2}, r_{2}}\left(\mathbb{R}^{n}\right)\right)_{\theta, r}=F_{q}^{s} L_{p, r}\left(\mathbb{R}^{n}\right)$.

Proof The operator $I$ in (3.4) is an isomorphic map of $F_{p_{k}, q}^{s}\left(\mathbb{R}^{n}\right)$ onto $f_{p_{k}, q}^{s}$. Hence, using the interpolation property and Theorem 3.5, we obtain that $I$ is an isomorphic map of $\left(F_{p_{1}, q}^{s}\left(\mathbb{R}^{n}\right), F_{p_{2}, q}^{s}\left(\mathbb{R}^{n}\right)\right)_{\theta, r}$ onto $f_{q}^{s} L_{p, r}$. Consequently, formula (a) follows from Theorem 3.3.

Then we derive formula (b) from (a) by applying the reiteration theorem for the real method (see [6, Theorem 3.11.5]).

\section{Some properties of Triebel-Lizorkin-Lorentz spaces}

By means of the interpolation formulae of Theorem 3.6 we can transfer to spaces $F_{q}^{s} L_{p, r}\left(\mathbb{R}^{n}\right)$ many other properties of the usual Triebel-Lizorkin spaces. For example, consider the Gauss-Weierstrass semi-group

$$
W_{t} f(x)=\frac{1}{(4 \pi t)^{n / 2}} \int_{\mathbb{R}^{n}} e^{-\frac{|x-y|^{2}}{4 t}} f(y) d y, f \in \mathcal{S}^{\prime}\left(\mathbb{R}^{n}\right), x \in \mathbb{R}^{n} .
$$

The following caloric smoothing holds. 
Theorem 4.1 Let $d \geq 0,0<p<\infty, s \in \mathbb{R}$ and $0<q, r \leq \infty$. Then there is $a$ constant $c>0$ such that for all $t$ with $0<t \leq 1$ and all $f \in F_{q}^{s} L_{p, r}\left(\mathbb{R}^{n}\right)$,

$$
t^{d / 2}\left\|W_{t} f\left|F_{q}^{s+d} L_{p, r}\left(\mathbb{R}^{n}\right)\|\leq c\| f\right| F_{q}^{s} L_{p, r}\left(\mathbb{R}^{n}\right)\right\| .
$$

Proof Let $0<p_{1}<p<p_{2}<\infty$ and $0<\theta<1$ such that $\frac{1}{p}=\frac{1-\theta}{p_{1}}+\frac{\theta}{p_{2}}$. Consider the operator $T_{t} f=t^{d / 2} W_{t} f$. By [56, Theorem 3.35], there is a constant $c_{1}>0$ such that for all $t$ with $0<t \leq 1$ and all $f \in F_{p_{j}, q}^{s}$ we have $\left\|T_{t} f\left|F_{p_{j}, q}^{s+d}\left(\mathbb{R}^{n}\right)\left\|\leq c_{1}\right\| f\right| F_{p_{j}, q}^{s}\left(\mathbb{R}^{n}\right)\right\|$. Whence, the result follows by interpolating the operator $T_{t}$ by the real method with parameter $(\theta, r)$ and using Theorem 3.6/(a).

We can also derive the following result on Fourier multipliers.

Theorem 4.2 Let $1<p<\infty, s \in \mathbb{R}$ and $0<q, r \leq \infty$. Then there are numbers $N \in \mathbb{N}$ and $c>0$ such that

$$
\left\|(m \hat{f})^{\vee}\left|F_{q}^{s} L_{p, r}\left(\mathbb{R}^{n}\right)\left\|\leq C \sup _{\substack{|\alpha| \leq N, x \in \mathbb{R}^{n}}}|x|^{|\alpha|}\left|D^{\alpha} m(x)\right| \cdot\right\| f\right| F_{q}^{s} L_{p, r}\left(\mathbb{R}^{n}\right)\right\| .
$$

Proof Let $\sigma>n\left(\max \left(\frac{1}{p}, 1\right)-1\right), 0<p<\infty$ and $0<q \leq \infty$. Then

$$
\left\|f\left|F_{p, q}^{\sigma}\left(\mathbb{R}^{n}\right)\|\sim\| f\right| L_{p}\left(\mathbb{R}^{n}\right)\right\|+\left\|f \mid \dot{F}_{p, q}^{\sigma}\left(\mathbb{R}^{n}\right)\right\|
$$

(see [49, Section 2.3.3]), where $\dot{F}_{p, q}^{\sigma}\left(\mathbb{R}^{n}\right)$ is the related homogeneous space. Let $T f=(m \hat{f})^{\vee}$. Using the corresponding multiplier assertion for the homogeneous spaces $\dot{F}_{p, q}^{s}\left(\mathbb{R}^{n}\right)$ [48, Theorem 5.2.2, p. 241] and the well-known Michlin-Hörmander Fourier multiplier theorem for $L_{p}\left(\mathbb{R}^{n}\right), 1<p<\infty$, we derive for $s>0$ that there are numbers $N$ and $c_{1}>0$ such that

$$
\left\|T f\left|F_{p, q}^{s}\left(\mathbb{R}^{n}\right)\left\|\leq c_{1} \sup _{\substack{|\alpha| \leq N, x \in \mathbb{R}^{n}}}|x|^{|\alpha|}\left|D^{\alpha} m(x)\right| \cdot\right\| f\right| F_{p, q}^{s}\left(\mathbb{R}^{n}\right)\right\| .
$$

We can extend this assertion from $s>0$ to $s \in \mathbb{R}$ with the help of the lift operator

$$
I_{\delta} f=\left(\left(1+|x|^{2}\right)^{-\delta / 2} \hat{f}\right)^{\vee}, \delta \in \mathbb{R},
$$

which maps $F_{p, q}^{s}\left(\mathbb{R}^{n}\right)$ isomorphically onto $F_{p, q}^{s+\delta}\left(\mathbb{R}^{n}\right)$ (see [48, Section 2.3.8]) and satisfies that $T=I_{-\delta} T I_{\delta}$. Finally, interpolating $T$ and using Theorem 3.6/(a) we conclude the wanted result.

Remark 4.3 In Theorem 4.2, any $N>n\left(\frac{1}{2}+\max \left(\frac{1}{p}, \frac{1}{q}\right)\right)$ is sufficient (but surely not optimal). See [54, p. 89] and the references given in there.

As it is pointed out in [49, Chapter 4] and [56, Section 2.2.1], in applications of spaces $A_{p, q}^{s}\left(\mathbb{R}^{n}\right), A \in\{B, F\}$, to problems of partial differential equations, for example to boundary value problems for elliptic PDEs, the following four properties are crucial: traces of $A_{p, q}^{s}\left(\mathbb{R}^{n}\right)$ on hyperplanes, invariance of $A_{p, q}^{s}\left(\mathbb{R}^{n}\right)$ with respect to diffeomorphisms of $\mathbb{R}^{n}$ onto itself, the existence of linear extension operators of 
the corresponding spaces $A_{p, q}^{s}\left(\mathbb{R}_{+}^{n}\right)$ on $\mathbb{R}_{+}^{n}$ to $A_{p, q}^{s}\left(\mathbb{R}^{n}\right)$, and several types of pointwise multipliers. It is natural to ask if these properties hold for Triebel-Lizorkin-Lorentz spaces. Three of these four key questions can be treated satisfactory using the interpolation properties of spaces $F_{q}^{s} L_{p, r}\left(\mathbb{R}^{n}\right)$. We write down the outcome below. Concerning traces we refer to [7].

A continuous one-to-one map of $\mathbb{R}^{n}$ onto itself,

$$
\begin{aligned}
& y=\psi(x)=\left(\psi_{1}(x), \ldots, \psi_{n}(x)\right), x \in \mathbb{R}^{n}, \\
& x=\psi^{-1}(y)=\left(\psi_{1}^{-1}(y), \ldots, \psi_{n}^{-1}(y)\right), y \in \mathbb{R}^{n},
\end{aligned}
$$

is called a diffeomorphism if all components $\psi_{j}(x)$ and $\psi_{j}^{-1}(y)$ are real $C^{\infty}$ functions on $\mathbb{R}^{n}$ and for $j=1, \ldots, n$,

$$
\sup _{x \in \mathbb{R}^{n}}\left(\left|D^{\alpha} \psi_{j}(x)\right|+\left|D^{\alpha} \psi_{j}^{-1}(x)\right|\right)<\infty \text { for all } \alpha \in \mathbb{N}_{0}^{n} \text { with }|\alpha|>0 .
$$

Then $\varphi \rightarrow \varphi \circ \psi$, given by $(\varphi \circ \psi)(x)=\varphi(\psi(x))$, is a one-to-one map of $\mathcal{S}\left(\mathbb{R}^{n}\right)$ onto itself. This can be extended to a one-to-one map of $\mathcal{S}^{\prime}\left(\mathbb{R}^{n}\right)$ onto itself as described in [56, Section 2.3]. As mentioned there,

$$
D_{\psi}: A_{p, q}^{s}\left(\mathbb{R}^{n}\right) \longrightarrow A_{p, q}^{s}\left(\mathbb{R}^{n}\right), D_{\psi} f=f \circ \psi
$$

is an isomorphic map for all spaces $A_{p, q}^{s}\left(\mathbb{R}^{n}\right), A \in\{B, F\}, s \in \mathbb{R}$ and $0<p, q \leq \infty$. Using Theorem 3.6/(a) this property can be extended to Triebel-Lizorkin-Lorentz spaces with the following outcome.

Theorem 4.4 Let $s \in \mathbb{R}, 0<p<\infty$ and $0<q, r \leq \infty$. Then

$$
D_{\psi}: F_{q}^{s} L_{p, r}\left(\mathbb{R}^{n}\right) \longrightarrow F_{q}^{s} L_{p, r}\left(\mathbb{R}^{n}\right), D_{\psi} f=f \circ \psi,
$$

is an isomorphic map in $F_{q}^{s} L_{p, r}\left(\mathbb{R}^{n}\right)$.

As for smooth multipliers, for $\rho \in \mathbb{R}$ consider the Hölder-Zygmund spaces $C^{\rho}\left(\mathbb{R}^{n}\right)=$ $B_{\infty, \infty}^{\rho}\left(\mathbb{R}^{n}\right)$. Using the corresponding assertion for $F_{p, q}^{s}\left(\mathbb{R}^{n}\right)[56$, Theorem 2.30] and again Theorem 3.6/(a) we obtain the following.

Theorem 4.5 Let $0<q, r \leq \infty, 0<p<\infty, s \in \mathbb{R}$ and $\rho>\max \left(s, n\left(\max \left\{\frac{1}{p}, 1\right\}-\right.\right.$ 1) -s). Then $g \in C^{\rho}\left(\mathbb{R}^{n}\right)$ is a pointwise multiplier for $F_{q}^{s} L_{p, r}\left(\mathbb{R}^{n}\right)$. Furthermore, there is a constant $c>0$ such that

$$
\left\|g f\left|F_{q}^{s} L_{p, r}\left(\mathbb{R}^{n}\right)\|\leq c\| g\right| C^{\rho}\left(\mathbb{R}^{n}\right)\right\| \cdot\left\|f \mid F_{q}^{s} L_{p, r}\left(\mathbb{R}^{n}\right)\right\|
$$

for all $g \in C^{\rho}\left(\mathbb{R}^{n}\right)$ and all $f \in F_{q}^{s} L_{p, r}\left(\mathbb{R}^{n}\right)$.

Next we let $\mathbb{R}_{+}^{n}=\left\{x=\left(x_{1}, \ldots, x_{n}\right) \in \mathbb{R}^{n}: x_{n}>0\right\}$. As usual, define $F_{q}^{s} L_{p, r}\left(\mathbb{R}_{+}^{n}\right)$ by restriction of $F_{q}^{s} L_{p, r}\left(\mathbb{R}^{n}\right)$ with

$$
\left\|f \mid F_{q}^{s} L_{p, r}\left(\mathbb{R}_{+}^{n}\right)\right\|=\inf \left\{\left\|g\left|F_{q}^{s} L_{p, r}\left(\mathbb{R}^{n}\right) \|: g\right|_{\mathbb{R}_{+}^{n}}=f\right\} .\right.
$$

The restriction operator re $f=\left.f\right|_{\mathbb{R}_{+}^{n}}$ defines a bounded linear map from $F_{q}^{s} L_{p, r}\left(\mathbb{R}^{n}\right)$ onto $F_{q}^{s} L_{p, r}\left(\mathbb{R}_{+}^{n}\right)$. We ask for a bounded linear operator ext,

$$
\text { ext : } F_{q}^{s} L_{p, r}\left(\mathbb{R}_{+}^{n}\right) \longrightarrow F_{q}^{s} L_{p, r}\left(\mathbb{R}^{n}\right),
$$

such that re $\circ$ ext $=\mathrm{id}$, where id is the identity map in $F_{q}^{s} L_{p, r}\left(\mathbb{R}_{+}^{n}\right)$. 
Theorem 4.6 Let $s \in \mathbb{R}, 0<p<\infty$ and $0<q, r \leq \infty$. There is a bounded linear (and even universal) extension operator from $F_{q}^{s} L_{p, r}\left(\mathbb{R}_{+}^{n}\right)$ to $F_{q}^{s} L_{p, r}\left(\mathbb{R}^{n}\right)$.

Proof Let $0<p_{1}<p<p_{2}<\infty$ and $0<\theta<1$ with $\frac{1}{p}=\frac{1-\theta}{p_{1}}+\frac{\theta}{p_{2}}$. Interpolating re $: F_{p_{j}, q}^{s}\left(\mathbb{R}^{n}\right) \longrightarrow F_{p_{j}, q}^{s}\left(\mathbb{R}_{+}^{n}\right)$ by the real method with parameters $(\theta, r)$ and using Theorem 3.6/(a), we get that

$$
\text { re }: F_{q}^{s} L_{p, r}\left(\mathbb{R}^{n}\right) \longrightarrow\left(F_{p_{1}, q}^{s}\left(\mathbb{R}_{+}^{n}\right), F_{p_{2}, q}^{s}\left(\mathbb{R}_{+}^{n}\right)\right)_{\theta, r}
$$

is bounded. This yields that

$$
F_{q}^{s} L_{p, r}\left(\mathbb{R}_{+}^{n}\right) \hookrightarrow\left(F_{p_{1}, q}^{s}\left(\mathbb{R}_{+}^{n}\right), F_{p_{2}, q}^{s}\left(\mathbb{R}_{+}^{n}\right)\right)_{\theta, r} .
$$

Besides, by the well-known assertions on extensions for the spaces $F_{p, q}^{s}\left(\mathbb{R}^{n}\right)$ (see [56, Section 2.5.2] and the references given there) there is a bounded linear operator ext : $F_{p_{j}, q}^{s}\left(\mathbb{R}_{+}^{n}\right) \longrightarrow F_{p_{j}, q}^{s}\left(\mathbb{R}^{n}\right), j=1,2$, such that re $\circ$ ext = id, the identity map in $F_{p_{j}, q}^{s}\left(\mathbb{R}_{+}^{n}\right)$. Using again the interpolation theorem and Theorem 3.6/(a), we obtain that

$$
\operatorname{ext}:\left(F_{p_{1}, q}^{s}\left(\mathbb{R}_{+}^{n}\right), F_{p_{2}, q}^{s}\left(\mathbb{R}_{+}^{n}\right)\right)_{\theta, r} \longrightarrow F_{q}^{s} L_{p, r}\left(\mathbb{R}^{n}\right)
$$

is bounded. Take any $f \in\left(F_{p_{1}, q}^{s}\left(\mathbb{R}_{+}^{n}\right), F_{p_{2}, q}^{s}\left(\mathbb{R}_{+}^{n}\right)\right)_{\theta, r}$. Then ext $f \in F_{q}^{s} L_{p, r}\left(\mathbb{R}^{n}\right)$ and so $f=\operatorname{re}(\operatorname{ext} f) \in F_{q}^{s} L_{p, r}\left(\mathbb{R}_{+}^{n}\right)$. Furthermore,

$$
\begin{aligned}
\left\|f \mid F_{q}^{s} L_{p, r}\left(\mathbb{R}_{+}^{n}\right)\right\| & \leq\left\|\operatorname{ext} f \mid F_{q}^{s} L_{p, r}\left(\mathbb{R}^{n}\right)\right\| \\
& \leq c\left\|f \mid\left(F_{p_{1}, q}^{s}\left(\mathbb{R}_{+}^{n}\right), F_{p_{2}, q}^{s}\left(\mathbb{R}_{+}^{n}\right)\right)_{\theta, r}\right\| .
\end{aligned}
$$

Consequently, $F_{q}^{s} L_{p, r}\left(\mathbb{R}_{+}^{n}\right)=\left(F_{p_{1}, q}^{s}\left(\mathbb{R}_{+}^{n}\right), F_{p_{2}, q}^{s}\left(\mathbb{R}_{+}^{n}\right)\right)_{\theta, r}$ with equivalence of quasinorms and

$$
\operatorname{ext}: F_{q}^{s} L_{p, r}\left(\mathbb{R}_{+}^{n}\right) \longrightarrow F_{q}^{s} L_{p, r}\left(\mathbb{R}^{n}\right)
$$

is bounded and satisfies that re $\circ$ ext $=\mathrm{id}$ in $F_{q}^{s} L_{p, r}\left(\mathbb{R}_{+}^{n}\right)$.

Similarly, let $\Omega$ be a domain (= non-empty open set) in $\mathbb{R}^{n}$ and define $F_{q}^{s} L_{p, r}(\Omega)$ by restriction. Proceeding as in Theorem 4.6 but using now the corresponding results on extensions for the spaces $F_{p, q}^{s}(\Omega)$ (see [56, Section 2.6.4]) one can derive the following.

Theorem 4.7 Let $s \in \mathbb{R}, 0<p<\infty, 0<q, r \leq \infty$ and let $\Omega$ be a bounded $C^{\infty}$ domain in $\mathbb{R}^{n}$. There is a bounded linear (and even universal) extension operator from $F_{q}^{s} L_{p, r}(\Omega)$ to $F_{q}^{s} L_{p, r}\left(\mathbb{R}^{n}\right)$.

Using [41, Theorem 2.2] (see also [50, Theorem 1.105]), we also have that Theorem 4.7 holds for bounded Lipschitz domains. 


\section{Multiplications}

As one can see in $[54,55]$, multiplication properties of Triebel-Lizorkin spaces are important for their applications to Navier-Stokes and nonlinear heat equations. Next we study the case of spaces $F_{q}^{s} L_{p, r}\left(\mathbb{R}^{n}\right)$.

We start with a preliminary result in the case $s=0$ which extends Hölder inequality.

Proposition 5.1 Let $1<p_{1}, p_{2}, p<\infty$ with $\frac{1}{p}=\frac{1}{p_{1}}+\frac{1}{p_{2}}$, let $0<q_{1}, q_{2} \leq 2 \leq q \leq \infty$ and $0<r_{1}, r_{2}, r \leq \infty$ with $\frac{1}{r}=\frac{1}{r_{1}}+\frac{1}{r_{2}}$. Then we have

$$
F_{q_{1}}^{0} L_{p_{1}, r_{1}}\left(\mathbb{R}^{n}\right) \cdot F_{q_{2}}^{0} L_{p_{2}, r_{2}}\left(\mathbb{R}^{n}\right) \hookrightarrow F_{q}^{0} L_{p, r}\left(\mathbb{R}^{n}\right)
$$

Proof Since $q_{j} \leq 2 \leq q$, it is clear from Definition 3.1 that

$$
F_{q_{j}}^{s} L_{p, r}\left(\mathbb{R}^{n}\right) \hookrightarrow F_{2}^{s} L_{p, r}\left(\mathbb{R}^{n}\right) \hookrightarrow F_{q}^{s} L_{p, r}\left(\mathbb{R}^{n}\right), j=1,2, s \in \mathbb{R} .
$$

Hence, by (3.1) and Hölder inequality for Lorentz spaces (2.7), we get

$$
\begin{aligned}
F_{q_{1}}^{0} L_{p_{1}, r_{1}}\left(\mathbb{R}^{n}\right) \cdot F_{q_{2}}^{0} L_{p_{2}, r_{2}}\left(\mathbb{R}^{n}\right) \hookrightarrow F_{2}^{0} L_{p_{1}, r_{1}}\left(\mathbb{R}^{n}\right) \cdot F_{2}^{0} L_{p_{2}, r_{2}}\left(\mathbb{R}^{n}\right) \\
\quad=L_{p_{1}, r_{1}}\left(\mathbb{R}^{n}\right) \cdot L_{p_{2}, r_{2}}\left(\mathbb{R}^{n}\right) \hookrightarrow L_{p, r}\left(\mathbb{R}^{n}\right) \hookrightarrow F_{q}^{0} L_{p, r}\left(\mathbb{R}^{n}\right) .
\end{aligned}
$$

Next we consider the case of spaces with positive smoothness $s$ and with differential dimension $(s-n / p)$ satisfying the same condition as in Proposition 5.1, that is, the sum of the differential dimension of the spaces in the source equal to the differential dimension of the space in the target. We put $\frac{1}{p^{s}}=\frac{1}{p}+\frac{s}{n}$ and we rely on the following result (see [18, Theorem 2.4.3, p. 52] or [40, Theorem 4.8.2/1, p. 238]): If $s>0$, $1<p_{1}, p_{2}, p<\infty$ with $\frac{1}{p}=\frac{1}{p_{1}}+\frac{1}{p_{2}}$ and $0<q \leq \infty$, then

$$
F_{p_{1}^{s}, q}^{s}\left(\mathbb{R}^{n}\right) \cdot F_{p_{2}^{s}, q}^{s}\left(\mathbb{R}^{n}\right) \hookrightarrow F_{p^{s}, q}^{s}\left(\mathbb{R}^{n}\right) .
$$

Theorem 5.2 Let $s>0,0<q, r_{1}, r_{2} \leq \infty$ and $1<p_{1}, p_{2}, p<\infty$ with $\frac{1}{p}=\frac{1}{p_{1}}+\frac{1}{p_{2}}$. Then

$$
F_{q}^{s} L_{p_{1}^{s}, r_{1}}\left(\mathbb{R}^{n}\right) \cdot F_{q}^{s} L_{p_{2}^{s}, r_{2}}\left(\mathbb{R}^{n}\right) \hookrightarrow F_{q}^{s} L_{p^{s}, r}\left(\mathbb{R}^{n}\right)
$$

where $\frac{1}{p_{1}^{s}}=\frac{1}{p_{1}}+\frac{s}{n}, \frac{1}{p_{2}^{s}}=\frac{1}{p_{2}}+\frac{s}{n}, \frac{1}{p^{s}}=\frac{1}{p}+\frac{s}{n}$ and $r \geq \min \left(r_{1}, r_{2}\right)$.

Proof Fix $p_{2}$. Given any $0<\varepsilon<\min \left(1, p_{1}-1, p_{2}-1\right)$, let $\delta=\varepsilon p_{2} /\left(p_{1}+p_{2}\right)$. Then

$$
\frac{1+\varepsilon}{p_{1}}+\frac{1}{p_{2}}=\frac{1+\delta}{p} \text { and } \frac{1-\varepsilon}{p_{1}}+\frac{1}{p_{2}}=\frac{1-\delta}{p} .
$$

Write

$$
u_{1}=\frac{p_{1}}{1+\varepsilon}, u_{2}=\frac{p_{1}}{1-\varepsilon}, v_{1}=\frac{p}{1+\delta} \text { and } v_{2}=\frac{p}{1-\delta}
$$


According to (5.1), there is a constant $c>0$ such that for any $g \in F_{p_{2}^{s}, q}^{s}\left(\mathbb{R}^{n}\right)$, the operator $T f=f g$ is bounded from $F_{u_{j}^{s}, q}^{s}\left(\mathbb{R}^{n}\right)$ into $F_{v_{j}^{s}, q}^{s}\left(\mathbb{R}^{n}\right)$ with norm less than or equal to $c$ for $j=1,2$. Since

$$
\frac{1 / 2}{u_{1}^{s}}+\frac{1 / 2}{u_{2}^{s}}=\frac{1}{p_{1}^{s}} \text { and } \frac{1 / 2}{v_{1}^{s}}+\frac{1 / 2}{v_{2}^{s}}=\frac{1}{p^{s}}
$$

using the interpolation theorem with parameters $\left(1 / 2, r_{1}\right)$ and Theorem $3.6 /(a)$, we derive that

$$
F_{q}^{s} L_{p_{1}^{s}, r_{1}}\left(\mathbb{R}^{n}\right) \cdot F_{p_{2}^{s}, q}^{s}\left(\mathbb{R}^{n}\right) \hookrightarrow F_{q}^{s} L_{p^{s}, r_{1}}\left(\mathbb{R}^{n}\right) .
$$

To complete the proof we keep now $p_{1}$ fixed and apply the same argument but using this time Theorem 3.6/(b) to identify the resulting spaces. This produces the target space $F_{q}^{s} L_{p^{s}, r_{2}}\left(\mathbb{R}^{n}\right)$. The last index $\left(r_{2}\right)$ can be improved to $r \geq \min \left(r_{1}, r_{2}\right)$ because we can start by fixing $p_{1}$ and then continue fixing $p_{2}$.

Now we turn our attention to cases where the target space has positive differential dimension.

Theorem 5.3 Assume that $0<p_{1}, p_{2}, p<\infty$ with $\max \left(\frac{1}{p_{1}}, \frac{1}{p_{2}}\right)<\frac{1}{p}<\frac{1}{p_{1}}+\frac{1}{p_{2}}$. Let $s>n\left(\frac{1}{p_{1}}+\frac{1}{p_{2}}-\frac{1}{p}\right)$ and $2 s>n \max \left(\frac{1}{p_{1}}+\frac{1}{p_{2}}-1,0\right)$. Let $0<q, r_{1}, r_{2} \leq \infty$ and $r \geq \min \left(r_{1}, r_{2}\right)$. Then

$$
F_{q}^{s} L_{p_{1}, r_{1}}\left(\mathbb{R}^{n}\right) \cdot F_{q}^{s} L_{p_{2}, r_{2}}\left(\mathbb{R}^{n}\right) \hookrightarrow F_{q}^{s} L_{p, r}\left(\mathbb{R}^{n}\right) .
$$

Proof According to [44, Theorem 4.4.1, p. 120] or [40, Theorem 4.8.2/2, p. 239], we have that

$$
F_{p_{1}, q}^{s}\left(\mathbb{R}^{n}\right) \cdot F_{p_{2}, q}^{s}\left(\mathbb{R}^{n}\right) \hookrightarrow F_{p, q}^{s}\left(\mathbb{R}^{n}\right) .
$$

Then the result follows by proceeding similarly as in the proof of the previous theorem and using Theorem 3.6.

In the paper by Hobus and Saal on Triebel-Lizorkin-Lorentz spaces and the NavierStokes equations, an important role is played by the following multiplication result (see [25, Lemma 6.4 and Remark 1.2]):

Let $s>0,1<p, q<\infty$ and $1 \leq r \leq \infty$. Then there exists $\bar{\varepsilon}>0$ such that for all $0<\varepsilon<\bar{\varepsilon}$ we have

$$
F_{q}^{s} L_{2 p-\varepsilon, r}\left(\mathbb{R}^{n}\right) \cdot F_{q}^{s} L_{2 p-\varepsilon, r}\left(\mathbb{R}^{n}\right) \hookrightarrow F_{q}^{s} L_{p, r}\left(\mathbb{R}^{n}\right) .
$$

Note that Theorem 5.3 allows to extend (5.3). Indeed, given any $s>0$ we may allow $n /(2 s+n)<p<\infty$ and $0<q, r \leq \infty$, because if

$$
\bar{\varepsilon}=\min \left(p, \frac{2 p^{2} s}{n+p s}, \frac{4 s p+2 n(p-1)}{2 s+n}\right),
$$

then for any $0<\varepsilon<\bar{\varepsilon}$, embedding (5.3) follows from Theorem 5.3.

According to [56, Section 2.7] if $0<p_{1} \leq p_{2}<\infty, s>n / p_{1}$ and $0<q \leq \infty$, one has

$$
F_{p_{2}, q}^{s}\left(\mathbb{R}^{n}\right) \cdot F_{p_{1}, q}^{s}\left(\mathbb{R}^{n}\right) \hookrightarrow F_{p_{1}, q}^{s}\left(\mathbb{R}^{n}\right) .
$$

With the help of Theorem 3.6 and proceeding in the same way as in Theorem 5.2 we can derive the following. 
Theorem 5.4 Let $0<p_{1}<p_{2}<\infty, 0<q \leq \infty, s>n / p_{1}$ and $r \geq \min \left(r_{1}, r_{2}\right)$. Then

$$
F_{q}^{s} L_{p_{2}, r_{2}}\left(\mathbb{R}^{n}\right) \cdot F_{q}^{s} L_{p_{1}, r_{1}}\left(\mathbb{R}^{n}\right) \hookrightarrow F_{q}^{s} L_{p_{1}, r}\left(\mathbb{R}^{n}\right) .
$$

The last result shows that any element of $F_{q}^{s} L_{p_{2}, r}\left(\mathbb{R}^{n}\right)$ is a pointwise multiplier for $F_{q}^{s} L_{p_{1}, r}\left(\mathbb{R}^{n}\right)$ with $0<p_{1}<p_{2}<\infty, s>n / p_{1}$ and $0<q, r \leq \infty$.

In contrast to (5.4), the preceding argument (and Theorem 5.4) does not cover the case $p_{1}=p_{2}$, which corresponds to multiplication algebra. However, using the bilinear interpolation theorem, we can establish the following.

Theorem 5.5 Let $0<p<\infty, s>n / p, 0<q \leq \infty, 0<r \leq \min (1, q)$ and $r<p$. Then $F_{q}^{s} L_{p, r}\left(\mathbb{R}^{n}\right)$ is a multiplication algebra.

Proof Take $r<p_{1}<p<p_{2}<\infty$ and $0<\theta<1$ such that $\frac{1}{p}=\frac{1-\theta}{p_{1}}+\frac{\theta}{p_{2}}$ and $s>n / p_{1}$. By (5.4), the bilinear operator $T(f, g)=f g$ is bounded acting from $F_{p_{j}, q}^{s}\left(\mathbb{R}^{n}\right) \times$ $F_{p_{j}, q}^{s}\left(\mathbb{R}^{n}\right)$ into $F_{p_{j}, q}^{s}\left(\mathbb{R}^{n}\right), j=1,2$. The space $F_{p_{j}, q}^{s}\left(\mathbb{R}^{n}\right)$ is $\min \left(1, p_{j}, q\right)$-Banach and so it is $r$-Banach. Since, by Theorem 3.6/(a), we have that $\left(F_{p_{1}, q}^{s}\left(\mathbb{R}^{n}\right), F_{p_{2}, q}^{s}\left(\mathbb{R}^{n}\right)\right)_{\theta, r}=$ $F_{q}^{s} L_{p, r}\left(\mathbb{R}^{n}\right)$, applying Theorem 2.2 we derive that

$$
T: F_{q}^{s} L_{p, r}\left(\mathbb{R}^{n}\right) \times F_{q}^{s} L_{p, r}\left(\mathbb{R}^{n}\right) \longrightarrow F_{q}^{s} L_{p, r}\left(\mathbb{R}^{n}\right) \text { boundedly. }
$$

In other words, $F_{q}^{s} L_{p, r}\left(\mathbb{R}^{n}\right)$ is a multiplicative algebra.

In particular, $F_{q}^{s} L_{p, 1}\left(\mathbb{R}^{n}\right)$ is both a Banach space and a multiplicative algebra for $1<p<\infty, s>n / p$ and $1 \leq q \leq \infty$. Otherwise, for these $p, s$ and $q$, if $1 \leq r_{1} \leq 2$ and $\frac{1}{r_{2}}=\frac{2}{r_{1}}-1$, then proceeding as in Theorem 5.5 we derive from Theorem 2.2 that

$$
F_{q}^{s} L_{p, r_{1}}\left(\mathbb{R}^{n}\right) \cdot F_{q}^{s} L_{p, r_{1}}\left(\mathbb{R}^{n}\right) \hookrightarrow F_{q}^{s} L_{p, r_{2}}\left(\mathbb{R}^{n}\right) .
$$

We have established Theorem 5.5 and (5.5) with the help of interpolation theory. Perhaps a more direct approach based on paramultiplication results (see [40]) might lead to improve these results. This idea and the property of multiplication algebra of $F_{p, q}^{s}\left(\mathbb{R}^{n}\right)$ for $s>n / p$ support:

Conjecture 5.6 Theorem 5.5 holds for all $0<r \leq \infty$.

Remark 5.7 Interpolation of Banach algebras is a question which has been of interest since the beginning of abstract interpolation theory with the contribution of Bishop [8] and Calderón [10]. See also the papers by Zafran [60], Kaijser [28] and Cobos, Fernández-Cabrera and Martínez [13].

The following embeddings will be useful for the last result of this section. As a consequence of Definition 3.1 we have that

$$
F_{q}^{s_{1}} L_{p, r}\left(\mathbb{R}^{n}\right) \hookrightarrow F_{q}^{s_{2}} L_{p, r}\left(\mathbb{R}^{n}\right), 0<p<\infty, 0<r \leq \infty, s_{2}<s_{1} .
$$

On the other hand, the well-known Jawerth-Franke embedding

$$
F_{p_{1}, q_{1}}^{s_{1}}\left(\mathbb{R}^{n}\right) \hookrightarrow F_{p_{2}, q_{2}}^{s_{2}}\left(\mathbb{R}^{n}\right), 0<p_{1}<p_{2}<\infty, s_{1}-\frac{n}{p_{1}}=s_{2}-\frac{n}{p_{2}}
$$

for all $0<q_{1}, q_{2} \leq \infty$ can be extended by using Theorem 3.6 to

$$
F_{q_{1}}^{s_{1}} L_{p_{1}, r}\left(\mathbb{R}^{n}\right) \hookrightarrow F_{q_{2}}^{s_{2}} L_{p_{2}, r}\left(\mathbb{R}^{n}\right), 0<r \leq \infty,
$$

(this last embedding is a special case of [43, Theorem 1.6]). 
Theorem 5.8 Let $0<p<\infty, s>\max (\sigma, n / p)$ and $0<q, r \leq \infty$. Then

$$
F_{q}^{s} L_{p, r}\left(\mathbb{R}^{n}\right) \cdot F_{q}^{s} L_{p, r}\left(\mathbb{R}^{n}\right) \hookrightarrow F_{q}^{\sigma} L_{p, r}\left(\mathbb{R}^{n}\right) .
$$

Proof Take $p<p_{2}<\infty$ such that $s-\frac{n}{p}=\sigma-\frac{n}{p_{2}}$. By (5.7) we have $F_{q}^{s} L_{p, r}\left(\mathbb{R}^{n}\right) \hookrightarrow$ $F_{q}^{\sigma} L_{p_{2}, r}\left(\mathbb{R}^{n}\right)$. Moreover, by (5.6), $F_{q}^{s} L_{p, r}\left(\mathbb{R}^{n}\right) \stackrel{p_{2}}{\hookrightarrow} F_{q}^{\sigma} L_{p, r}\left(\mathbb{R}^{n}\right)$. Consequently, according to Theorem 5.4, we obtain

$$
F_{q}^{s} L_{p, r}\left(\mathbb{R}^{n}\right) \cdot F_{q}^{s} L_{p, r}\left(\mathbb{R}^{n}\right) \hookrightarrow F_{q}^{\sigma} L_{p_{2}, r}\left(\mathbb{R}^{n}\right) \cdot F_{q}^{\sigma} L_{p, r}\left(\mathbb{R}^{n}\right) \hookrightarrow F_{q}^{\sigma} L_{p, r}\left(\mathbb{R}^{n}\right) .
$$

\section{Besov-Lorentz spaces}

Let $\left(\varphi_{k}\right)_{k \in \mathbb{N}_{0}}$ be the smooth dyadic resolution of unity constructed in Section 3 from the generating function $\varphi_{0} \in \mathcal{S}\left(\mathbb{R}^{n}\right)$.

Definition 6.1 Let $s \in \mathbb{R}, 0<p<\infty$ and $0<q, r \leq \infty$. The Besov-Lorentz space $B_{q}^{s} L_{p, r}\left(\mathbb{R}^{n}\right)$ collects all $f \in \mathcal{S}^{\prime}\left(\mathbb{R}^{n}\right)$ having a finite quasi-norm

$$
\left\|f \mid B_{q}^{s} L_{p, r}\left(\mathbb{R}^{n}\right)\right\|=\left(\sum_{k=0}^{\infty} 2^{k s q}\left\|\left(\varphi_{k} \hat{f}\right)^{\vee} \mid L_{p, r}\left(\mathbb{R}^{n}\right)\right\|^{q}\right)^{1 / q}
$$

(with the usual modification if $q=\infty$ ).

Definition 6.1 extends [47, Definition 2.4.1/(a), p. 181] to the complete range of the parameters. Besov-Lorentz spaces are denoted by $B_{p, q,(r)}^{s}\left(\mathbb{R}^{n}\right)$ in [47] and by $B_{q}^{s}\left[L^{p, r}\right]$ in [43]. Clearly, when $r=p$, the space $B_{q}^{s} L_{p, p}\left(\mathbb{R}^{n}\right)$ coincides with the usual Besov space $B_{p, q}^{s}\left(\mathbb{R}^{n}\right)$. Spaces $B_{q}^{s} L_{p, r}\left(\mathbb{R}^{n}\right)$ are quasi-Banach spaces (Banach spaces if $p>1$ and $\min (q, r) \geq 1)$.

Almeida and Caetano [3] have introduced Besov-Lorentz spaces but replacing in Definition 6.1 the role of $L_{p, r}\left(\mathbb{R}^{n}\right)$ by the local Hardy-Lorentz space $h_{p, r}$ which is defined by

$$
h_{p, r}=\left\{f \in \mathcal{S}^{\prime}\left(\mathbb{R}^{n}\right):\left\|f\left|h_{p, r}\|=\| \sup _{0<t<1}\right|(\psi(t \cdot) \hat{f})^{\vee}|| L_{p, r}\left(\mathbb{R}^{n}\right)\right\|<\infty\right\} .
$$

Here $\psi \in \mathcal{S}\left(\mathbb{R}^{n}\right)$ is compactly supported and $\psi(x)=1$ if $|x| \leq 1$. The space $h_{p, r}$ does not depend on the particular choice of $\psi$ (see [3, Theorem 3.1]). Clearly, $h_{p, p}$ coincides with the usual local Hardy space $h_{p}$ (see $\left.[22,48]\right)$. We designate by $B_{q}^{s} h_{p, r}\left(\mathbb{R}^{n}\right)$ the Besov-Lorentz spaces of Almeida and Caetano.

According to [3, Theorems 4.3 and 4.7] we have with equivalence of quasi-norms

$$
\begin{array}{r}
\left(h_{p_{1}}, h_{p_{2}}\right)_{\theta, r}=h_{p, r} \quad \text { provided that } 0<p_{1} \neq p_{2}<\infty, 0<r<\infty, \\
0<\theta<1 \text { and } \frac{1}{p}=\frac{1-\theta}{p_{1}}+\frac{\theta}{p_{2}},
\end{array}
$$

and

$$
h_{p, r}=L_{p, r}\left(\mathbb{R}^{n}\right) \quad \text { provided that } 1<p<\infty \text { and } 0<r<\infty \text {. }
$$


Putting together (6.1), [48, Theorem 2.5.8/1] and Theorem 3.6, for $0<p_{1}<p<$ $p_{2}<\infty, 0<\theta<1, \frac{1}{p}=\frac{1-\theta}{p_{1}}+\frac{\theta}{p_{2}}$ and $0<r<\infty$, we get

$$
h_{p, r}=\left(h_{p_{1}}, h_{p_{2}}\right)_{\theta, r}=\left(F_{p_{1}, 2}^{0}\left(\mathbb{R}^{n}\right), F_{p_{2}, 2}^{0}\left(\mathbb{R}^{n}\right)\right)_{\theta, r}=F_{2}^{0} L_{p, r}\left(\mathbb{R}^{n}\right) \text {. }
$$

Equality $h_{p, r}=F_{2}^{0} L_{p, r}\left(\mathbb{R}^{n}\right)$ can also be derived proceeding similarly to [48, Theorem 2.5.8/1].

Note that (6.2) yields that $B_{q}^{s} L_{p, r}\left(\mathbb{R}^{n}\right)=B_{q}^{s} h_{p, r}\left(\mathbb{R}^{n}\right)$ provided that $1<p<\infty$ and $0<r<\infty$. In order to show that they also coincide when $0<p \leq 1$, we first extend to Lorentz spaces several results of [48] for Lebesgue spaces.

For $\Omega$ a compact subset of $\mathbb{R}^{n}$, we put

$$
\mathcal{S}^{\Omega}\left(\mathbb{R}^{n}\right)=\left\{\varphi \mid \varphi \in \mathcal{S}\left(\mathbb{R}^{n}\right) \text { and } \operatorname{supp} \hat{\varphi} \subseteq \Omega\right\}
$$

and for $0<p<\infty$ and $0<r \leq \infty$, we write

$$
L_{p, r}^{\Omega}\left(\mathbb{R}^{n}\right)=\left\{f \mid f \in \mathcal{S}^{\prime}\left(\mathbb{R}^{n}\right), \operatorname{supp} \hat{f} \subseteq \Omega \text { and }\left\|f \mid L_{p, r}\left(\mathbb{R}^{n}\right)\right\|<\infty\right\} .
$$

As usual, if $1 \leq p \leq \infty$ we put $\frac{1}{p}+\frac{1}{p^{\prime}}=1$.

Lemma 6.2 Let $\Omega$ be a compact subset of $\mathbb{R}^{n}$ and let $0<p<\infty$ and $0<r \leq \infty$. There is a constant $c>0$ such that

$$
\left\|\varphi\left|L_{\infty}\left(\mathbb{R}^{n}\right)\|\leq c\| \varphi\right| L_{p, r}\left(\mathbb{R}^{n}\right)\right\| \text { for any } \varphi \in \mathcal{S}^{\Omega}\left(\mathbb{R}^{n}\right) .
$$

Furthermore,

$$
L_{p, r}^{\Omega}\left(\mathbb{R}^{n}\right) \hookrightarrow L_{\infty}^{\Omega}\left(\mathbb{R}^{n}\right) .
$$

Proof Let $\psi \in \mathcal{S}\left(\mathbb{R}^{n}\right)$ such that $\hat{\psi}(x)=1$ if $x \in \Omega$. Then

$$
|\varphi(x)|=\left|(\hat{\varphi})^{\vee}(x)\right|=\left|(\hat{\psi} \hat{\varphi})^{\vee}(x)\right|=c|\psi * \varphi(x)|=c\left|\int_{\mathbb{R}^{n}} \psi(x-y) \varphi(y) d y\right| .
$$

Take any $0<s<\min (p, r, 1)$. Using Hölder inequality for Lorentz spaces (2.7), we obtain

$$
\begin{aligned}
|\varphi(x)| & \leq c \int_{\mathbb{R}^{n}}|\varphi(y)|^{1-s}|\varphi(y)|^{s}|\psi(x-y)| d y \\
& \leq c \sup _{y \in \mathbb{R}^{n}}|\varphi(y)|^{1-s} \int_{\mathbb{R}^{n}}|\varphi(y)|^{s}|\psi(x-y)| d y \\
& \leq c \sup _{y \in \mathbb{R}^{n}}|\varphi(y)|^{1-s}\left\||\varphi|^{s}\left|L_{p / s, r / s}\left(\mathbb{R}^{n}\right)\|\|\right| \psi\right\| L_{(p / s)^{\prime},(r / s)^{\prime}}\left(\mathbb{R}^{n}\right) \| \\
& =c_{\Omega} \sup _{y \in \mathbb{R}^{n}}|\varphi(y)|^{1-s}\left\|\varphi \mid L_{p, r}\left(\mathbb{R}^{n}\right)\right\|^{s} .
\end{aligned}
$$

This yields (6.4).

To establish (6.5) we proceed as in [48, Theorem 1.4.1/Step 1]. Let $\varphi \in \mathcal{S}\left(\mathbb{R}^{n}\right)$ with $\varphi(0)=1$ and supp $\hat{\varphi} \subseteq\{y|| y \mid \leq 1\}$. For $f \in L_{p, r}^{\Omega}\left(\mathbb{R}^{n}\right)$ and $0<\delta<1$, we 
write $f_{\delta}(x)=\varphi(\delta x) f(x)$. Then $f_{\delta} \in \mathcal{S}^{B}\left(\mathbb{R}^{n}\right)$ where $B$ is a closed ball, centered at the origin, such that

$$
\{y \mid \text { there is } x \in \Omega \text { with }|x-y| \leq 1\} \subseteq B,
$$

and $f_{\delta}$ converges to $f$ in $L_{\infty}\left(\mathbb{R}^{n}\right)$ as $\delta \downarrow 0$ (see [48, pp.22-23]). By (6.4) we get

$$
\begin{aligned}
\left\|f_{\delta} \mid L_{\infty}\left(\mathbb{R}^{n}\right)\right\| & \leq c\left\|f_{\delta}\left|L_{p, r}\left(\mathbb{R}^{n}\right)\|=c\| \varphi(\delta \cdot) f\right| L_{p, r}\left(\mathbb{R}^{n}\right)\right\| \\
& \leq c\left\|\varphi\left|L_{\infty}\left(\mathbb{R}^{n}\right)\|\| f\right| L_{p, r}\left(\mathbb{R}^{n}\right)\right\| \leq c_{1}\left\|f \mid L_{p, r}\left(\mathbb{R}^{n}\right)\right\| .
\end{aligned}
$$

Therefore, letting $\delta \downarrow 0$ we conclude that

$$
\left\|f\left|L_{\infty}\left(\mathbb{R}^{n}\right)\left\|\leq c_{1}\right\| f\right| L_{p, r}\left(\mathbb{R}^{n}\right)\right\| .
$$

Next we establish a Fourier multiplier result for Lorentz spaces.

Theorem 6.3 Let $\Omega$, $\Gamma$ be compact subsets of $\mathbb{R}^{n}$. Let $0<p<\infty, 0<r \leq \infty$ and put

$$
\sigma= \begin{cases}1 & \text { if } \min (p, r)>1 \\ \min (p, r) / 2 & \text { if } \min (p, r) \leq 1 .\end{cases}
$$

Then there is a constant $c=c(\Omega, \Gamma, p, r)>0$ such that for any $f \in L_{p, r}^{\Omega}\left(\mathbb{R}^{n}\right)$ and $\check{M} \in L_{\sigma}^{\Gamma}\left(\mathbb{R}^{n}\right)$ the inequality

$$
\left\|(M \hat{f})^{\vee}\left|L_{p, r}\left(\mathbb{R}^{n}\right)\|\leq c\| \check{M}\right| L_{\sigma}\left(\mathbb{R}^{n}\right)\right\|\left\|f \mid L_{p, r}\left(\mathbb{R}^{n}\right)\right\|
$$

holds.

Proof We know by Lemma 6.2 that $f \in L_{\infty}^{\Omega}\left(\mathbb{R}^{n}\right)$. If $\min (p, r)>1$, since

$$
(M \hat{f})^{\vee}(x)=c_{1} \int_{\mathbb{R}^{n}} \check{M}(y) f(x-y) d y=c_{1} \check{M} * f(x), x \in \mathbb{R}^{n},
$$

using the properties of the convolution in Lorentz spaces, that is (2.8), we get

$$
\left\|(M \hat{f})^{\vee}\left|L_{p, r}\left(\mathbb{R}^{n}\right)\left\|=c_{1}\right\| \check{M} * f\right| L_{p, r}\left(\mathbb{R}^{n}\right)\right\| \leq c\left\|\check{M}\left|L_{1}\left(\mathbb{R}^{n}\right)\|\| f\right| L_{p, r}\left(\mathbb{R}^{n}\right)\right\| .
$$

Suppose now that $\min (p, r) \leq 1$. This time the assumption is $|\check{M}|^{\sigma} \in L_{1}\left(\mathbb{R}^{n}\right)$ and so for each $x \in \mathbb{R}^{n}$ we have $\check{M}(\cdot) f(x-\cdot) \in L_{\sigma}\left(\mathbb{R}^{n}\right)$. Moreover, $\operatorname{supp}(\check{M}(\cdot) f(x-\cdot))^{\wedge} \subseteq$ $\Gamma-\Omega$ (see [48, pp. 25-26]). It follows from [48, 1.4.1/(3)] that $\check{M}(\cdot) f(x-\cdot) \in L_{1}\left(\mathbb{R}^{n}\right)$ and

$$
\begin{aligned}
\left|(M \hat{f})^{\vee}(x)\right|^{\sigma} & =c_{2}\left|\int_{\mathbb{R}^{n}} \check{M}(y) f(x-y) d y\right|^{\sigma} \\
& \leq c_{3} \int_{\mathbb{R}^{n}}|\check{M}(y) f(x-y)|^{\sigma} d y \\
& =c_{3}|\check{M}|^{\sigma} *|f|^{\sigma}(x) .
\end{aligned}
$$


Consequently, using (2.8), we conclude that

$$
\begin{aligned}
\left\|(M \hat{f})^{\vee} \mid L_{p, r}\left(\mathbb{R}^{n}\right)\right\| & =\left\|\left|(M \hat{f})^{\vee}\right|^{\sigma} \mid L_{p / \sigma, r / \sigma}\left(\mathbb{R}^{n}\right)\right\|^{1 / \sigma} \\
& \leq c 4\left\||\check{M}|^{\sigma} *|f|^{\sigma} \mid L_{p / \sigma, r / \sigma}\left(\mathbb{R}^{n}\right)\right\|^{1 / \sigma} \\
& \leq c\left\|\left.|\check{M}|^{\sigma}\left|L_{1}\left(\mathbb{R}^{n}\right)\left\|^{1 / \sigma}\right\|\right| f\right|^{\sigma} \mid L_{p / \sigma, r / \sigma}\left(\mathbb{R}^{n}\right)\right\|^{1 / \sigma} \\
& =c\left\|\check{M}\left|L_{\sigma}\left(\mathbb{R}^{n}\right)\|\| f\right| L_{p, r}\left(\mathbb{R}^{n}\right)\right\| .
\end{aligned}
$$

This completes the proof.

Now we are ready to establish that both kinds of Besov spaces are equal.

Theorem 6.4 Let $-\infty<s<\infty, 0<q \leq \infty$ and $0<p, r<\infty$. Then we have with equivalence of quasi-norms

$$
B_{q}^{s} L_{p, r}\left(\mathbb{R}^{n}\right)=B_{q}^{s} h_{p, r}\left(\mathbb{R}^{n}\right)
$$

Proof Let $\left(\varphi_{k}\right)_{k=0}^{\infty}$ be a smooth dyadic resolution of unity. It is enough to show that for any $f \in \mathcal{S}^{\prime}\left(\mathbb{R}^{n}\right)$ we have

$$
\left\|\left(\varphi_{k} \hat{f}\right)^{\vee}\left|L_{p, r}\left(\mathbb{R}^{n}\right)\|\sim\|\left(\varphi_{k} \hat{f}\right)^{\vee}\right| h_{p, r}\right\| .
$$

Let $\psi \in \mathcal{S}\left(\mathbb{R}^{n}\right)$ compactly supported and with $\psi(x)=1$ if $|x| \leq 1$. For $t \leq 2^{-(k+1)}$ we have that $\psi(t \cdot) \varphi_{k}=\varphi_{k}$ because supp $\varphi_{k} \subseteq\left\{x: 2^{k-1} \leq|x| \leq 2^{k+1}\right\}$. Therefore,

$$
\begin{aligned}
\left\|\left(\varphi_{k} \hat{f}\right)^{\vee} \mid L_{p, r}\left(\mathbb{R}^{n}\right)\right\| & =\left\|\left(\psi\left(2^{-(k+1)} \cdot\right) \varphi_{k} \hat{f}\right)^{\vee} \mid L_{p, r}\left(\mathbb{R}^{n}\right)\right\| \\
& \leq\left\|\sup _{0<t<1}\left|\left(\psi(t \cdot) \varphi_{k} \hat{f}\right)^{\vee}\right|\left|L_{p, r}\left(\mathbb{R}^{n}\right)\|=\|\left(\varphi_{k} \hat{f}\right)^{\vee}\right| h_{p, r}\right\| .
\end{aligned}
$$

To check the converse inequality note that

$$
\operatorname{supp} \varphi_{0} \subseteq\left\{x \in \mathbb{R}^{n}:|x| \leq 2\right\}, \varphi_{1}(x)=\varphi_{0}\left(2^{-1} x\right)-\varphi_{0}(x)
$$

and

$$
\varphi_{k}(x)=\varphi_{0}\left(2^{-k} x\right)-\varphi_{0}\left(2^{-k+1} x\right)=\varphi_{1}\left(2^{-k+1} x\right), k=2,3, \ldots
$$

Let $\left(\psi_{k}\right)_{k=0}^{\infty}$ be another smooth dyadic resolution of unity. Then analogous relationships hold among $\psi_{0}, \psi_{1}$ and $\psi_{k}$. According to (6.3) for $k=2,3, \ldots$ we get

$$
\begin{aligned}
\left\|\left(\varphi_{k} \hat{f}\right)^{\vee} \mid h_{p, r}\right\| & \sim\left\|\left(\varphi_{k} \hat{f}\right)^{\vee} \mid F_{2}^{0} L_{p, r}\left(\mathbb{R}^{n}\right)\right\| \\
& =\left\|\left(\sum_{m=0}^{\infty}\left|\psi_{m} \varphi_{k} \hat{f}(x)\right|^{2}\right)^{1 / 2} \mid L_{p, r}\left(\mathbb{R}^{n}\right)\right\| \\
& =\left\|\left(\sum_{j=-1}^{1}\left|\left(\psi_{k+j} \varphi_{k} \hat{f}\right)^{\vee}(x)\right|^{2}\right)^{1 / 2} \mid L_{p, r}\left(\mathbb{R}^{n}\right)\right\| \\
& \lesssim \sum_{j=-1}^{1}\left\|\left(\psi_{k+j} \varphi_{k} \hat{f}\right)^{\vee} \mid L_{p, r}\left(\mathbb{R}^{n}\right)\right\| .
\end{aligned}
$$


Moreover,

$$
\begin{aligned}
\left(\psi_{k+j} \varphi_{k} \hat{f}\right)^{\vee}(\xi) & =\left(\psi_{1}\left(2^{-k-j+1} \cdot\right) \varphi_{1}\left(2^{-k+1} \cdot\right) \hat{f}\right)^{\vee}(\xi) \\
& =2^{(k-1) n}\left(\psi_{1}\left(2^{-j} \cdot\right) \varphi_{1} \hat{f}\left(2^{k-1} \cdot\right)\right)^{\vee}\left(2^{k-1} \xi\right) .
\end{aligned}
$$

Hence

$$
\begin{aligned}
\left\|\left(\psi_{k+j} \varphi_{k} \hat{f}\right)^{\vee} \mid L_{p, r}\left(\mathbb{R}^{n}\right)\right\| & =2^{(k-1) n}\left\|\left(\psi_{1}\left(2^{-j} \cdot\right) \varphi_{1} \hat{f}\left(2^{k-1} \cdot\right)\right)^{\vee}\left(2^{k-1} \cdot\right) \mid L_{p, r}\left(\mathbb{R}^{n}\right)\right\| \\
& \leq 2^{(k-1) n(1-(1 / p))}\left\|\left(\psi_{1}\left(2^{-j} \cdot\right) \varphi_{1} \hat{f}\left(2^{k-1} \cdot\right)\right)^{\vee} \mid L_{p, r}\left(\mathbb{R}^{n}\right)\right\| \\
& =2^{(k-1) n(1-(1 / p))}\left\|\left(\psi_{1}\left(2^{-j} \cdot\right) \hat{h}\right)^{\vee} \mid L_{p, r}\left(\mathbb{R}^{n}\right)\right\|
\end{aligned}
$$

where $h=\left(\varphi_{1} \hat{f}\left(2^{k-1} \cdot\right)\right)^{\vee}$ and we have used that

$$
\left\|f(\lambda \cdot)\left|L_{p, r}\left(\mathbb{R}^{n}\right)\left\|=\lambda^{-n / p}\right\| f\right| L_{p, r}\left(\mathbb{R}^{n}\right)\right\| \quad \text { for } \lambda>0 .
$$

Since $j=-1,0,1$, we have

$$
\operatorname{supp} \psi_{1}\left(2^{-j}\right) \subseteq\{x:|x| \leq 8\} .
$$

Furthermore,

$$
\operatorname{supp} \hat{h}=\operatorname{supp} \varphi_{1} \hat{f}\left(2^{k+1} \cdot\right) \subseteq \operatorname{supp} \varphi_{1} \subseteq\{x:|x| \leq 4\} .
$$

So, the supports of $\psi_{1}\left(2^{-j}.\right)$ and $\hat{h}$ are contained in compact sets which are independent of $j$ and $k$. Applying Theorem 6.3, we obtain that

$$
\begin{aligned}
& \left\|\left(\psi_{k+j} \varphi_{k} \hat{f}\right)^{\vee}\left|L_{p, r}\left(\mathbb{R}^{n}\right)\left\|\leq 2^{(k-1) n(1-(1 / p))}\right\|\left(\psi_{1}\left(2^{-j} \cdot\right) \hat{h}\right)^{\vee}\right| L_{p, r}\left(\mathbb{R}^{n}\right)\right\| \\
& \lesssim 2^{(k-1) n(1-(1 / p))}\left\|\psi_{1}\left(2^{-j} \cdot\right)^{\vee}\left|L_{\sigma}\left(\mathbb{R}^{n}\right)\|\|\left(\varphi_{1} \hat{f}\left(2^{k-1} \cdot\right)\right)^{\vee}\right| L_{p, r}\left(\mathbb{R}^{n}\right)\right\| .
\end{aligned}
$$

We have $\psi_{1}\left(2^{-j} \cdot\right)^{\vee}=2^{j n} \check{\psi}_{1}\left(2^{j} \cdot\right)$ and

$$
\begin{aligned}
\left(\varphi_{1} \hat{f}\left(2^{k-1} \cdot\right)\right)^{\vee} & =2^{-(k-1) n}\left(\varphi_{1}\left(2^{-(k-1)} \cdot\right) \hat{f}\right)^{\vee}\left(2^{-(k-1)} \cdot\right) \\
& =2^{-(k-1) n}\left(\varphi_{k} \hat{f}\right)^{\vee}\left(2^{-(k-1)} \cdot\right) .
\end{aligned}
$$

Therefore

$$
\begin{aligned}
&\left\|\left(\psi_{k+j} \varphi_{k} \hat{f}\right)^{\vee} \mid L_{p, r}\left(\mathbb{R}^{n}\right)\right\| \\
& \lesssim 2^{(k-1) n(1-(1 / p))}\left\|\check{\psi}_{1}\left|L_{\sigma}\left(\mathbb{R}^{n}\right)\left\|2^{-(k-1) n}\right\|\left(\varphi_{k} \hat{f}\right)^{\vee}\left(2^{-(k-1)} \cdot\right)\right| L_{p, r}\left(\mathbb{R}^{n}\right)\right\| \\
& \lesssim\left\|\left(\varphi_{k} \hat{f}\right)^{\vee} \mid L_{p, r}\left(\mathbb{R}^{n}\right)\right\| .
\end{aligned}
$$

This yields that

$$
\left\|\left(\varphi_{k} \hat{f}\right)^{\vee}\left|h_{p, r}\|\lesssim\|\left(\varphi_{k} \hat{f}\right)^{\vee}\right| L_{p, r}\left(\mathbb{R}^{n}\right)\right\| .
$$

The cases $k=0,1$ can be treated similarly. The proof is complete.

We can now establish the following interpolation result which extends [47, Theorem 2.4.1/(c)] to the whole range of parameters (see also [3, Theorem 4.10]). 
Theorem 6.5 Let $0<\theta<1,-\infty<s_{1}, s_{2}<\infty, s=(1-\theta) s_{1}+\theta s_{2}, 0<p_{1} \neq p_{2}<\infty$, $\frac{1}{p}=\frac{1-\theta}{p_{1}}+\frac{\theta}{p_{2}}, 0<q_{1}, q_{2}<\infty$ and $\frac{1}{q}=\frac{1-\theta}{q_{1}}+\frac{\theta}{q_{2}}$. Then we have with equivalent quasi-norms

$$
\left(B_{p_{1}, q_{1}}^{s_{1}}\left(\mathbb{R}^{n}\right), B_{p_{2}, q_{2}}^{s_{2}}\left(\mathbb{R}^{n}\right)\right)_{\theta, q}=B_{q}^{s} L_{p, q}\left(\mathbb{R}^{n}\right) .
$$

Proof Let $\left(\varphi_{k}\right)_{k=0}^{\infty}$ be a smooth dyadic resolution of unity. Put $\tilde{\varphi}_{k}=\varphi_{k-1}+\varphi_{k}+\varphi_{k+1}$ with $\varphi_{-1} \equiv 0$. For $f \in \mathcal{S}^{\prime}\left(\mathbb{R}^{n}\right)$, put $J f=\left(\left(\varphi_{k} \hat{f}\right)^{\vee}\right)$ and for $\left(f_{k}\right) \in \ell_{q_{j}}^{s_{j}}\left(h_{p_{j}}\right)$ write $R\left(f_{k}\right)=\sum_{k=0}^{\infty}\left(\tilde{\varphi}_{k} \hat{f}_{k}\right)^{\vee}$ (convergence in $\mathcal{S}^{\prime}\left(\mathbb{R}^{n}\right)$ ). Then

$$
J: B_{p_{j}, q_{j}}^{s_{j}}\left(\mathbb{R}^{n}\right) \longrightarrow \ell_{q_{j}}^{s_{j}}\left(h_{p_{j}}\right) \text { and } R: \ell_{q_{j}}^{s_{j}}\left(h_{p_{j}}\right) \longrightarrow B_{p_{j}, q_{j}}^{s_{j}}\left(\mathbb{R}^{n}\right)
$$

are bounded operators and $R(J f)=f$. That is, $B_{p_{j}, q_{j}}^{s_{j}}\left(\mathbb{R}^{n}\right)$ is a retract of $\ell_{q_{j}}^{s_{j}}\left(h_{p_{j}}\right)$. The space $\ell_{q_{j}}^{s_{j}}\left(h_{p_{j}}\right)$ coincides with the vector-valued $\ell_{q_{j}}$-space modelled on the sequence of quasi-Banach spaces $\left(2^{s_{j} k} h_{p_{j}}\right)_{k \in \mathbb{N}_{0}}$. Hence, according to (2.3) and (6.1), we have

$$
\begin{aligned}
\left(\ell_{q_{1}}^{s_{1}}\left(h_{p_{1}}\right), \ell_{q_{2}}^{s_{2}}\left(h_{p_{2}}\right)\right)_{\theta, q} & =\left(\ell_{q_{1}}\left(2^{s_{1} k} h_{p_{1}}\right), \ell_{q_{2}}\left(2^{s_{2} k} h_{p_{2}}\right)\right)_{\theta, q} \\
& =\ell_{q}\left(2^{s k} h_{p, q}\right)=\ell_{q}^{s}\left(h_{p, q}\right) .
\end{aligned}
$$

Then [47, Theorem 1.2.4] and Theorem 6.4 yield that $\left(B_{p_{1}, q_{1}}^{s_{1}}\left(\mathbb{R}^{n}\right), B_{p_{2}, q_{2}}^{s_{2}}\left(\mathbb{R}^{n}\right)\right)_{\theta, q}=$ $B_{q}^{s} L_{p, q}\left(\mathbb{R}^{n}\right)$.

The precise description of $\left(B_{p_{1}, q_{1}}^{s_{1}}\left(\mathbb{R}^{n}\right), B_{p_{2}, q_{2}}^{s_{2}}\left(\mathbb{R}^{n}\right)\right)_{\theta, r}$ for $r \neq q$ with $\frac{1}{q}=\frac{1-\theta}{q_{1}}+\frac{\theta}{q_{2}}$ seems to be an open problem since Peetre stated it in his monograph on Besov spaces $[37$, p. 110].

If $p_{1}=p_{2}$ then we have the following formula.

Theorem 6.6 Let $-\infty<s_{1} \neq s_{2}<\infty, 0<p, r<\infty$ and $0<q_{1}, q_{2}, q \leq \infty$. Let $0<\theta<1$ and $s=(1-\theta) s_{1}+\theta s_{2}$. Then we have with equivalent quasi-norms

$$
\left(B_{q_{1}}^{s_{1}} L_{p, r}\left(\mathbb{R}^{n}\right), B_{q_{2}}^{s_{2}} L_{p, r}\left(\mathbb{R}^{n}\right)\right)_{\theta, q}=B_{q}^{s} L_{p, r}\left(\mathbb{R}^{n}\right) .
$$

Proof We proceed as in the proof of the previous theorem but using now that, according to (2.2), we have

$$
\left(\ell_{q_{1}}^{s_{1}}\left(h_{p, r}\right), \ell_{q_{2}}^{s_{2}}\left(h_{p, r}\right)\right)_{\theta, q}=\ell_{q}^{s}\left(h_{p, r}\right)
$$

The interpolation properties of Triebel-Lizorkin-Lorentz spaces (Theorem 3.6) have allowed us to transfer a number of properties of usual Triebel-Lizorkin spaces to spaces $F_{q}^{s} L_{p, r}\left(\mathbb{R}^{n}\right)$. However, for Besov-Lorentz spaces Theorem 6.5 is not exactly the counterpart of Theorem 3.6 because of the relationship among $q_{1}, q_{2}$ and $q$. The reason for the lack of a counterpart is that there is no reasonable generalization of the Lions-Peetre formula

$$
\left(L_{p_{1}}\left(A_{1}\right), L_{p_{2}}\left(A_{2}\right)\right)_{\theta, q}=L_{q}\left(\left(A_{1}, A_{2}\right)_{\theta, q}\right), \frac{1}{q}=\frac{1-\theta}{p_{1}}+\frac{\theta}{p_{2}} .
$$

See [16] (see also [21] and [12, Section 3]).

To overcome this obstruction we are going to characterize $B_{q}^{s} L_{p, r}\left(\mathbb{R}^{n}\right)$ as an interpolation space between Triebel-Lizorkin-Lorentz spaces. 
Theorem 6.7 Let $-\infty<s_{1} \neq s_{2}<\infty, 0<p, r<\infty$ and $0<q_{1}, q_{2}, q \leq \infty$. Let $0<\theta<1$ and $s=(1-\theta) s_{1}+\theta s_{2}$. Then we have with equivalent quasi-norms

$$
\left(F_{q_{1}}^{s_{1}} L_{p, r}\left(\mathbb{R}^{n}\right), F_{q_{2}}^{s_{2}} L_{p, r}\left(\mathbb{R}^{n}\right)\right)_{\theta, q}=B_{q}^{s} L_{p, r}\left(\mathbb{R}^{n}\right) .
$$

Proof For $j=1,2$, let $0<\tau_{j}, v_{j}<\infty$ such that

$$
\begin{cases}\tau_{j} \leq \min \left(p, q_{j}, r\right) & \text { for } p \neq q_{j} \text { and for } p=q_{j} \geq r, \\ \tau_{j}<p & \text { for } p=q_{j}<r,\end{cases}
$$

and

$$
\begin{cases}v_{j} \geq \max \left(p, q_{j}, r\right) & \text { for } p \neq q_{j} \text { and for } p=q_{j} \leq r, \\ v_{j}>p & \text { for } p=q_{j}>r .\end{cases}
$$

According to the sharp embeddings obtained by Seeger and Trebels [43, Theorems 1.1 and 1.2 ] we have

$$
B_{\tau_{j}}^{s_{j}} L_{p, r}\left(\mathbb{R}^{n}\right) \hookrightarrow F_{q_{j}}^{s_{j}} L_{p, r}\left(\mathbb{R}^{n}\right) \hookrightarrow B_{v_{j}}^{s_{j}} L_{p, r}\left(\mathbb{R}^{n}\right), j=1,2 .
$$

Consequently, applying Theorem 6.6, we derive

$$
\begin{aligned}
B_{q}^{s} L_{p, r}\left(\mathbb{R}^{n}\right) & =\left(B_{\tau_{1}}^{s_{1}} L_{p, r}\left(\mathbb{R}^{n}\right), B_{\tau_{2}}^{s_{2}} L_{p, r}\left(\mathbb{R}^{n}\right)\right)_{\theta, q} \\
& \hookrightarrow\left(F_{q_{1}}^{s_{1}} L_{p, r}\left(\mathbb{R}^{n}\right), F_{q_{2}}^{s_{2}} L_{p, r}\left(\mathbb{R}^{n}\right)\right)_{\theta, q} \\
& \hookrightarrow\left(B_{v_{1}}^{s_{1}} L_{p, r}\left(\mathbb{R}^{n}\right), B_{v_{2}}^{s_{2}} L_{p, r}\left(\mathbb{R}^{n}\right)\right)_{\theta, q}=B_{q}^{s} L_{p, r}\left(\mathbb{R}^{n}\right) .
\end{aligned}
$$

Since spaces $F_{q}^{s} L_{p, r}\left(\mathbb{R}^{n}\right)$ are independent of the generating function $\varphi_{0}$ of the resolution of unity, it follows from Theorem 6.7 that spaces $B_{q}^{s} L_{p, r}\left(\mathbb{R}^{n}\right)$ are also independent of $\varphi_{0}$ (with equivalent quasi-norms).

\section{Some properties of Besov-Lorentz spaces}

Now with the help of Theorem 6.7 we can transfer the properties established in the previous sections for Triebel-Lizorkin-Lorentz spaces to Besov-Lorentz spaces. We fix the outcome. As usual, spaces $B_{q}^{s} L_{p, r}\left(\mathbb{R}_{+}^{n}\right)$ and $B_{q}^{s} L_{p, r}(\Omega)$ are defined by restriction.

Theorem 7.1 Let $s \in \mathbb{R}, 0<p, r<\infty$ and $0<q \leq \infty$.

(i) If $\psi$ is a diffeomorphism in $\mathbb{R}^{n}$ then

$$
D_{\psi}: B_{q}^{s} L_{p, r}\left(\mathbb{R}^{n}\right) \longrightarrow B_{q}^{s} L_{p, r}\left(\mathbb{R}^{n}\right), D_{\psi} f=f \circ \psi,
$$

is an isomorphic map in $B_{q}^{s} L_{p, r}\left(\mathbb{R}^{n}\right)$.

(ii) Let $\rho>\max \left(s, n\left(\max \left(\frac{1}{p}, 1\right)-1\right)-s\right)$. Then

$$
\left\|g f\left|B_{q}^{s} L_{p, r}\left(\mathbb{R}^{n}\right)\|\leq c\| g\right| C^{\rho}\left(\mathbb{R}^{n}\right)\right\| \cdot\left\|f \mid B_{q}^{s} L_{p, r}\left(\mathbb{R}^{n}\right)\right\|
$$

for all $g \in C^{\rho}\left(\mathbb{R}^{n}\right)$ and all $f \in B_{q}^{s} L_{p, r}\left(\mathbb{R}^{n}\right)$. 
(iii) There is a bounded linear extension operator ext,

$$
\text { ext }: B_{q}^{s} L_{p, r}\left(\mathbb{R}_{+}^{n}\right) \longrightarrow B_{q}^{s} L_{p, r}\left(\mathbb{R}^{n}\right)
$$

Furthermore,

$$
\text { re o ext }=i d \text {, identity in } B_{q}^{s} L_{p, r}\left(\mathbb{R}_{+}^{n}\right)
$$

(iv) If $\Omega$ is a bounded $C^{\infty}$ domain in $\mathbb{R}^{n}$, there is a bounded linear extension operator

$$
\text { ext : } B_{q}^{s} L_{p, r}(\Omega) \longrightarrow B_{q}^{s} L_{p, r}\left(\mathbb{R}^{n}\right) \text {. }
$$

Furthermore,

$$
\text { re o ext }=i d \text {, identity in } B_{q}^{s} L_{p, r}(\Omega) \text {. }
$$

Property (iv) also holds for bounded Lipschitz domains.

Our next result shows sufficient conditions on the parameters for a Besov-Lorentz space to be a multiplication algebra.

Theorem 7.2 Let $0<p<\infty, s>n / p, 0<q \leq r \leq 1$ and $r<p$. Then $B_{q}^{s} L_{p, r}\left(\mathbb{R}^{n}\right)$ is a multiplication algebra.

Proof Take $r<p_{1}<p<p_{2}<\infty, \frac{n}{p_{1}}<s_{1}<s<s_{2}<\infty$ and $0<\tau, \theta<1$ with $\frac{1}{p}=\frac{1-\tau}{p_{1}}+\frac{\tau}{p_{2}}$ and $s=(1-\theta) s_{1}+\theta s_{2}$. According to Theorem 3.6 we have that

$$
F_{r}^{s_{j}} L_{p, r}\left(\mathbb{R}^{n}\right)=\left(F_{p_{1}, r}^{s_{j}}\left(\mathbb{R}^{n}\right), F_{p_{2}, r}^{s_{j}}\left(\mathbb{R}^{n}\right)\right)_{\tau, r} .
$$

Since spaces $F_{p_{j}, r}^{s_{j}}\left(\mathbb{R}^{n}\right)$ are $r$-Banach, it follows from Lemma 2.1 that $F_{r}^{s_{j}} L_{p, r}\left(\mathbb{R}^{n}\right)$ is also $r$-Banach. Consider now the bilinear operator $T(f, g)=f g$. By Theorem 5.5, the restrictions

$$
T: F_{r}^{s_{j}} L_{p, r}\left(\mathbb{R}^{n}\right) \times F_{r}^{s_{j}} L_{p, r}\left(\mathbb{R}^{n}\right) \longrightarrow F_{r}^{s_{j}} L_{p, r}\left(\mathbb{R}^{n}\right), j=1,2,
$$

are bounded. Moreover, by Theorem 6.7, we have

$$
\left(F_{r}^{s_{1}} L_{p, r}\left(\mathbb{R}^{n}\right), F_{r}^{s_{2}} L_{p, r}\left(\mathbb{R}^{n}\right)\right)_{\theta, q}=B_{q}^{s} L_{p, r}\left(\mathbb{R}^{n}\right) .
$$

Consequently, applying Theorem 2.2, we conclude that

$$
B_{q}^{s} L_{p, r}\left(\mathbb{R}^{n}\right) \cdot B_{q}^{s} L_{p, r}\left(\mathbb{R}^{n}\right) \hookrightarrow B_{q}^{s} L_{p, r}\left(\mathbb{R}^{n}\right) .
$$

In particular, if $1<p<\infty, s>n / p$ and $0<q \leq 1$, the space $B_{q}^{s} L_{p, 1}\left(\mathbb{R}^{n}\right)$ is a multiplication algebra.

In a similar way to the case of Triebel-Lizorkin-Lorentz spaces, the results above together with the fact that usual Besov spaces are multiplication algebras if $s>n / p$ (see [53, Theorem 1.16, p. 12]) support the following.

Conjecture 7.3 Spaces $B_{q}^{s} L_{p, r}\left(\mathbb{R}^{n}\right)$ are multiplication algebras for $0<p<\infty, s>$ $n / p$ and $0<q, r \leq \infty$.

We close the paper by recalling that the characterization of $B_{q}^{s} L_{p, q}\left(\mathbb{R}^{n}\right)$ in terms of wavelets has been established by Almeida [1, Corollary 3.2].

Acknowledgements Part of the research of Blanca F. Besoy was done while she visited the Institut für Mathematik of the Friedrich-Schiller-Universität Jena. She would like to thank the Group on Function Spaces of this Institut for the hospitality given to her during the visit.

The authors would like to thank the referee for his/her remarks. 


\section{Conflict of interest}

The authors declare that they have no conflict of interest.

Data sharing not applicable to this article as no datasets were generated or analysed during the current study.

\section{References}

1. Almeida, A.: On interpolation properties of generalized Besov spaces. In: Further Progress in Analysis, pp. 601-610. World Scientific, London (2009)

2. Almeida, A., Caetano, A.M.: Generalized Hardy Spaces. Acta Math. Sin. (Engl. Ser.) 26, 1673-1692 (2010)

3. Almeida, A., Caetano, A.: Real interpolation of generalized Besov-Hardy spaces and applications. J. Fourier Anal. Appl. 17, 691-719 (2011)

4. Aoki, T: Locally bounded linear topologycal maps. Proc. Imp. Acad. Tokyo 18, 588-594 (1942)

5. Bennett, C., Sharpley, R.: Interpolation of operators. Academic Press, Boston (1988)

6. Bergh, J., Löfström, J.: Interpolation spaces. An introduction. Springer, Berlin (1976)

7. Besoy, B.F., Haroske, D.D., Triebel, H: Traces of some weighted function spaces and related nonstandard real interpolation of Besov spaces. Preprint, Jena, 2020.

8. Bishop, E.A.: Holomorphic completion, analytic continuation, and the interpolation of seminorms Ann. Math. 78, 468-500 (1963)

9. Caetano, A.M.: Subatomic representation of Bessel potential spaces modelled on Lorentz spaces. In: The J.A. Sampaio Martins Anniversary Volume, Textos Mat. Sér. B, vol. 34, pp. 37âĂŞ47. Univ. Coimbra, Coimbra (2004)

10. Calderón, A.P.: Intermediate spaces and interpolation, the complex method. Studia Math. 24, 113-190 (1964)

11. Cianchi, A., Pick, L.: An optimal endpoint trace embedding. Ann. Inst. Fourier 60, 939-951 (2010)

12. Cobos, F.: On the type of interpolation spaces and $S_{p, q}$. Math. Nachr. 113, 59-64 (1983)

13. Cobos, F., Fernández-Cabrera, L.M., Martínez, A.: On interpolation of Banach algebras and factorization of weakly compact homomorphisms. Bull. Sci. Math. 130, 637-645 (2006)

14. Cobos, F., Fernández-Cabrera, L.M., Martínez, A.: Interpolation of compact bilinear operators among quasi-Banach spaces and applications. Math. Nachr. 291, 2168-2187 (2018)

15. Cobos, F., Martín, J.: On interpolation of function spaces by methods defined by means of polygons. J. Approx. Theory 132, 182-203 (2005)

16. Cwikel, M.: On $\left(L^{p_{0}}\left(A_{0}\right), L^{p_{1}}\left(A_{1}\right)\right)_{\theta, q}$. Proc. Amer. Math. Soc. 44, 286-292 (1974)

17. Edmunds, D.E., Evans,W.D.: Hardy operators, function spaces and embeddings. Springer, Berlin (2004)

18. Edmunds, D.E., Triebel, H.: Function spaces, entropy numbers, differential operators. Cambridge Univ. Press, Cambridge (1996)

19. Fefferman, C., Riviere, N.M., Sagher, Y.: Interpolation between $H^{p}$ spaces: The real method. Trans Amer. Math. Soc. 191, 75-81 (1974)

20. Fernández-Cabrera, L.M., Martínez, A.: Real interpolation of compact bilinear operators. J. Fourier Anal. Appl. 24, 1181-1203 (2018)

21. Freitag, D.: Reelle Interpolation zwischen $\ell_{p}$-Räumen mit Gewichten. Math. Nachr. 77, 101-115 (1977)

22. Goldberg, D.: A local version of real Hardy spaces. Duke Math. J. 46, 27-42 (1979)

23. Grafakos, L.: Classical Fourier analysis. 2nd ed., Springer, New York (2008)

24. Grafakos, L., Slavíková, L.: A sharp version of the Hörmander multiplier theorem. Int. Math. Res. Not. IMRN 15, 4764-4783 (2019)

25. Hobus, P., Saal, J.: Triebel-Lizorkin-Lorentz spaces and the Navier-Stokes equations. Z. Anal. Anwendungen 38, 41-72 (2019) 
26. Hunt, R.A.: On $L(p, q)$ spaces. Enseign. Math. 12, 249-276 (1966)

27. Janson, S.: On interpolation of multi-linear operators. In: Cwikel, M. et al. (eds.) Function spaces and applications, Lecture Notes in Math. vol. 1302, pp. 290-302. Springer, Berlin (1988)

28. Kaijser, S.: Interpolation of Banach algebras and open sets. Integral Equations Oper. Theory 41, 189-222 (2001)

29. Kalton, N.J.: Linear operators on $L_{p}$ for $0<p<1$. Trans. Amer. Math. Soc. 259, 319-355 (1980)

30. Karadzhov, G.E.: The interpolation method of "means" for quasinormed spaces, Doklady Acad. Nauk SSSR 209, 33-36 (1973)

31. Ko,H., Lee, S.: Fourier transform and regularity of characteristic functions. Proc. Amer. Math. Soc. 145, 1097-1107 (2017)

32. Köthe, G.: Topological Vector Spaces I. Springer, Berlin (1969)

33. König, H.: On the tensor stability of $s$-number ideals. Math. Ann. 269, 77-93 (1984)

34. Krée, P.: Interpolation d'espaces vectoriels qui ne sont ni normés, ni complets. Applications. Ann. Inst. Fourier 17, 137-174 (1967)

35. Lions, J.L., Peetre, J.: Sur une classe d'espaces d'interpolation. Inst. Hautes Études Sci. Publ. Math. 19, 5-68 (1964)

36. O’Neil, R.: Convolution operators and $L(p, q)$ spaces. Duke Math. J. 30, 129-142 (1963)

37. Peetre, J.: New thoughts on Besov spaces. Duke Univ. Math. Series, Duke Univ., Durham (1976)

38. Pietsch, A.: Operator ideals. North-Holland, Amsterdam (1980)

39. Rolewicz, S.: On a certain class of linear metric spaces. Bull. Acad. Polon. Sci. Sér. Sci. Math. Astrono. Phys. 5, 471-473 (1957)

40. Runst,T., Sickel, W.: Sobolev spaces of fractional order, Nemytskij operators, and nonlinear partial differential equations. W. de Gruyter, Berlin (1996)

41. Rychkov, V.S.: On restrictions and extensions of the Besov and Triebel-Lizorkin spaces with respect to Lipschitz domains. J. London Math. Soc. 60, 237-257 (1999)

42. Sagher, Y.: Interpolation of $r$-Banach spaces. Studia Math. 41, 45-70 (1972)

43. Seeger, A., Trebels, W.: Embeddings for spaces of Lorentz-Sobolev type. Math. Ann. 373, 1017-1056 (2019)

44. Sickel,W., Triebel, H.: Hölder inequalities and sharp embeddings in function spaces of $B_{p q}^{s}$ and $F_{p q}^{S}$ type. Z. Anal. Anwendungen 14, 105-140 (1995)

45. Stein, E.M.: Editor's note: the differentiability of functions in $\mathbb{R}^{n}$. Ann. of Math. 113, 383-385 (1981)

46. Torchinsky, A.: Real variable methods in harmonic analysis. Academic Press, San Diego (1986)

47. Triebel, H.: Interpolation theory, function spaces, differential operators. North-Holland, Amsterdam (1978)

48. Triebel, H.: Theory of function spaces. Birkhäuser, Basel (1983)

49. Triebel, H.: Theory of function spaces II. Birkhäuser, Basel (1992)

50. Triebel, H.: Theory of function spaces III. Birkhäuser, Basel (2006)

51. Triebel, H.: Function spaces and wavelets on domains. European Math. Soc. Publishing House, Zürich (2008)

52. Triebel, H.: Wavelet bases in Lorentz and Zygmund spaces. Georgian Math. J. 15, 389-402 (2008)

53. Triebel, H.: Local function spaces, heat and Navier-Stokes equations. European Math. Soc. Publishing House, Zürich (2013)

54. Triebel, H.: Hybrid function spaces, heat and Navier-Stokes equations. European Math. Soc. Publishing House, Zürich (2014)

55. Triebel, H.: PDE models for chemotaxis and hydrodynamics in supercritical function spaces. European Math. Soc. Publishing House, Zürich (2017)

56. Triebel, H.: Theory of function spaces IV. Birkhäuser, Basel (2020)

57. Xiang, Z., Yan, W.: On the well-posedness of the quasi-geostrophic equation in the Triebel-LizorkinLorentz spaces. J. Evol. Equ. 11, 241-263 (2011)

58. Xiang, Z., Yan, W.: On the well-posedness of Boussinesq equation in the Triebel-Lizorkin-Lorentz spaces. Abstract and Applied Analysis 2012 (2012), article ID 573084, 17p.

59. Yang, Q., Cheng, Z., Peng, L.: Uniform characterization of function spaces by wavelets. Acta Math Sci. Ser. A (Chin. Ed.) 25, 130-144 (2005)

60. Zafran, M.: The dichotomy problem for homogeneous Banach algebras. Ann. Math. 108, 97-105 (1978) 